\title{
An analytical and numerical study of axisymmetric flow around spheroids
}

\author{
By CHIEN-CHENG CHANG, BIING-HORNG LIOU \\ AND RUEY-LING CHERN \\ Institute of Applied Mechanics, College of Engineering, National Taiwan University, \\ Taipei 10764, Taiwan, R.O.C.
}

(Received 20 July 1990 and in revised form 11 May 1991)

Axisymmetric viscous flow around ellipsoids of circular section is studied in detail by the method of matched asymptotic expansion and a deterministic hybrid vortex method. The main feature of the hybrid vortex method consists in solving the viscous vorticity equation by interlacing a finite-difference method for diffusion and a vortex-in-cell method for convection and stretching. Numerical results are presented for an ellipsoid of axis ratio 2:1 and the limiting case of a sphere at various Reynolds numbers between 100 and 3000 . Special consideration is given to the evaluation of the drag coefficient which is computed with three different approaches, denoted by $\mathrm{S}, \mathrm{P}$ and $\mathrm{V}$ respectively. Particular emphasis is placed on the numerical implications and physical significance of each of these different approaches. Comparisons between numerical and analytical results at small times show very close agreement in each case. Separation angles, wake lengths and stationary drag coefficients for the sphere are also found to be in good agreement with previous results by a finite-difference method and with the standard drag curve.

\section{Introduction}

For many years there has been considerable effort directed to the study of viscous flows around a sphere or an ellipsoid under various flow conditions. This is not only because of the intrinsic interest of these flows but also because of their important practical applications. Analytical works have been mainly restricted to flows at fairly low Reynolds numbers. Typical papers in this area are Chester \& Breach (1969), Oliver \& Chung (1985) and Sano (1981) for flow around a sphere, and Kim \& Arunachalam (1987) for flow around an ellipsoid. Van Dyke (1975) contains further references. Steady-state solutions are the common interest of these papers (except that of Sano). Numerical analysis of flow around a sphere has been carried out for a wide range of Reynolds numbers; references include Hamielec, Hoffman \& Ross (1967), Rimon \& Cheng (1969), LeClair, Hamielec \& Pruppacher (1970) and Dennis \& Walker (1972). There seems to be lack of work on flow around ellipsoids at moderate Reynolds numbers. The present paper aims to provide a combined analytical and numerical study of axisymmetric viscous flow around ellipsoids of circular section by asymptotic analysis and by a hybrid vortex method.

Numerical results are presented for an ellipsoid of axis ratio $2: 1$ and the limiting case of a sphere at various Reynolds numbers between 100 and 3000 . Special consideration is given to the evaluation of the drag coefficient which is computed with three approaches, denoted by $S, P$ and $V$ respectively. Approach $S$ is the most traditional, employing vorticity and its normal derivative on the surface. Approach 


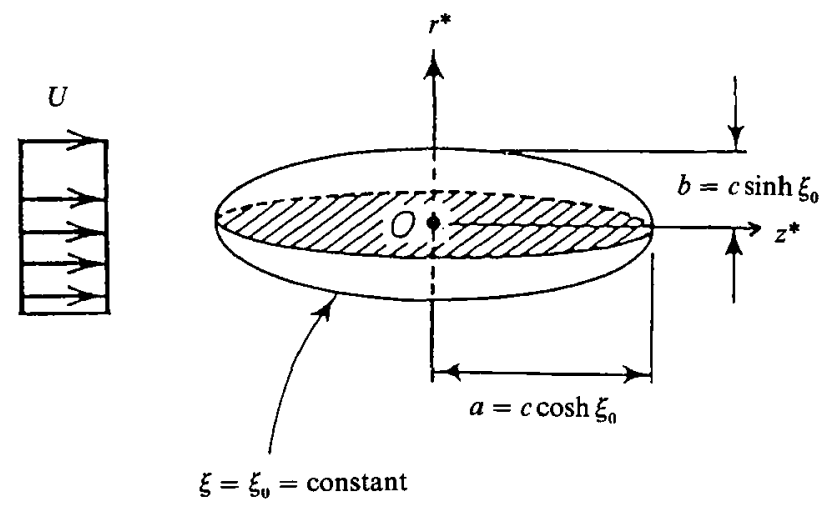

Figure 1. Schematic of the physical problem.

$\mathrm{P}$ involves integrating the momentum equation from infinity to the body surface, while $\mathrm{V}$ requires evaluating the product of the velocity and vorticity in the flow and the surface vorticity. Approach $V$ provides a very interesting physical explanation of how the flow contributes to the drag coefficient through the vorticity within the flow and on the body surface. The present analysis indicates that either $\mathrm{P}$ or $\mathrm{V}$ is superior to $S$ in the early stages of flow development. Comparisons between numerical and analytical results at small times show very satisfactory agreement for each case. Separation angles, wake lengths and stationary drag coefficients are also found to be in good agreement with the finite-difference results of Rimon \& Cheng (1969) and with the standard drag curve.

The present asymptotic analysis is standard, and is somewhat generalized to include the case of an accelerating ellipsoid. Similar analysis has been employed, among others, by Bar-Lev \& Yang (1975) for flow around a circular cylinder, by Wang (1967) for flow around an elliptic cylinder, and by Peace \& Riley (1983) for a pair of vortices impinging on a flat plate. The asymptotic analysis is by its nature valid only at small times; flow behaviour at relatively long times is analysed by a deterministic hybrid vortex method. Previous investigations by Chang (1989) and Chang \& Chern (1991) have shown some success of the deterministic method for flows around a circular cylinder. The method is here extended to study axisymmetric flow around ellipsoids of circular section. The main feature of the hybrid method consists in solving the viscous vorticity equation by interlacing a finite-difference method for diffusion and a vortex-in-cell method for convection and stretching. The hybrid method belongs to the general class of methods under the heading 'computational methods with vortices', which have been of great interest during past decades; several methods of this kind, especially those of stochastic content, have been critically reviewed by Sarpkaya (1989). It has to be emphasized that the present method does not resolve all the difficulties mentioned by Sarpkaya. Nevertheless, for unsteady laminar flows, the present approach appears to be quantitatively reliable. It is noted that even though axisymmetry is assumed, the flow is three-dimensional in nature in that the effect of vortex stretching is present. Moreover, the hybrid vortex method maintains the same simplicity as its two-dimensional version since the Helmholtz theorem holds in both two and three space dimensions. 


\section{Basic equations}

Consider a fluid of density $\rho$ and constant kinematic viscosity $\nu$. An ellipsoid is accelerated from rest to a constant final velocity $U_{0}^{*}$. The flow around the ellipsoid is assumed to be governed by the Navier-Stokes and the continuity equations. Some flow parameters are shown in figure 1 . Let the reference length and velocity be respectively one half of the focal length $(c)$ and the final speed of the distant flow $\left(U_{0}^{*}\right)$. At any instant, we take the inertial frame moving with the velocity of the ellipsoid; the equations of motion are invariant under such a (Galilean) transformation. In dimensionless form, these equations may be written

$$
\begin{gathered}
\frac{\partial u}{\partial t}+(u \cdot \nabla) u=-\nabla P+\frac{2}{R e} \nabla^{2} u, \\
\nabla \cdot u=0,
\end{gathered}
$$

where Reynolds number $R e=2 U_{0}^{*} c / \nu, u$ denotes the velocity and $P$ pressure. The dimensional velocity $u^{*}$, time $t^{*}$ and pressure $P^{*}$ are given by

$$
u^{*}=U_{0}^{*} u, \quad t^{*}=c t / U_{0}^{*}, \quad P^{*}=\rho U_{0}^{* 2} P .
$$

Another useful definition of the Reynolds number is $R e_{d}=2 U_{0}^{*} a / \nu$, with $2 a$ the chord length of the ellipsoid. Similarly we may define $t_{d}$ by $t^{*}=a t_{d} / U_{0}^{*}$. Notice that since we are concerned with axisymmetric flow, it is adequate to introduce the dimensionless stream function $\psi=\psi(r, z)$ such that

$$
\boldsymbol{u}=\boldsymbol{\nabla} \times\left(\frac{\psi}{r} \boldsymbol{e}_{\phi}\right) \equiv \boldsymbol{\nabla} \times \boldsymbol{B}
$$

The equation of continuity is therefore automatically satisfied. The vorticity $\omega$ has only one component, i.e. $\omega=\omega e_{\phi}$, and is related to $u$ through $\omega=\nabla \times u$. In the stream function-vorticity formulation, the equations of motion are recast as

$$
\nabla^{2} B=-\omega
$$

and

$$
\frac{\partial \omega}{\partial t}+(u \cdot \nabla) \omega=\omega \cdot \nabla u+\frac{2}{R e} \nabla^{2} \omega
$$

Most of our analysis is based on a spheroidal coordinate system $(\xi, \theta)$, defined by

$$
z^{*}+\mathrm{i} r^{*}=c \cosh (\xi+\mathrm{i} \theta),
$$

where $2 c$ is the focal length. The surface of the ellipsoid is represented by the constant coordinate $\xi=\xi_{0}$ while the chord length is $2 a=2 c \cosh \xi_{0}$. The ratio of the major axis to the minor axis is $R=1 / \tanh \xi_{0}$ while the eccentricity is given by $e=\operatorname{sech} \xi_{0}$. In terms of the coordinates, the vorticity equation becomes

$$
\frac{\partial \omega}{\partial t}+\frac{1}{h}\left(u_{\xi} \frac{\partial \omega}{\partial \xi}+u_{\theta} \frac{\partial \omega}{\partial \theta}\right)=\frac{\omega}{h}\left(\operatorname{coth} \xi u_{\xi}+\cot \theta u_{\theta}\right)+\frac{2}{R e}\left(\nabla^{2} \omega-\frac{\omega}{\sinh ^{2} \xi \sin ^{2} \theta}\right)
$$

where $h^{2}=\cosh ^{2} \xi-\cos ^{2} \theta$ and $\nabla^{2}=h^{-2}\left(\partial_{\xi}^{2}+\partial_{\theta}^{2}+\operatorname{coth} \xi \partial_{\xi}+\cot \theta \partial_{\theta}\right)$. The velocity components $u_{\xi}$ and $u_{\theta}$ are related to $\psi$ through

$$
u_{\xi}=\frac{1}{h \sinh \xi \sin \theta} \frac{\partial \psi}{\partial \theta}, \quad u_{\theta}=-\frac{1}{h \sinh \xi \sin \theta} \frac{\partial \psi}{\partial \xi} .
$$


Similarly, (2.5) becomes

$$
-\omega=\frac{1}{\sinh \xi \sin \theta}\left(\nabla^{2} \psi-2 \frac{\operatorname{coth} \xi}{h^{2}} \frac{\partial \psi}{\partial \xi}-2 \frac{\cot \theta}{h^{2}} \frac{\partial \psi}{\partial \theta}\right) .
$$

At the surface of the ellipsoid, the boundary conditions can be chosen to be

$$
\psi=\frac{\partial \psi}{\partial \xi}=0 \quad \text { at } \quad \xi=\xi_{0} .
$$

Far away from the ellipsoid, a uniform parallel flow with velocity $U$ is approaching the ellipsoid; the far-field conditions are therefore given by

$$
\frac{1}{h \sinh \xi \sin \theta} \frac{\partial \psi}{\partial \xi}=U \sin \theta \quad \text { as } \quad \xi \rightarrow \infty .
$$

Notice that $t$ enters $U$ as a parameter rather than a variable. At any instant, we look at the flow in an inertial frame.

\subsection{Drag coefficients}

The drag coefficient is defined by

$$
C_{D}=\frac{D^{*}}{\frac{1}{2} \rho \pi U_{0}^{* 2} a^{2}}=\frac{2 D^{*}}{\rho \pi U_{0}^{* 2} c^{2} \cosh ^{2} \xi_{0}}
$$

where $D^{*}$ is the total drag exerted on the ellipsoid. The drag coefficient consists of two parts: one due to pressure and one due to friction, i.e. $C_{D}=C_{D P}+C_{D F}$. Let $S_{0}$ denote the surface of the ellipsoid. Under our choice of coordinate system, the drag coefficient is given by $C_{D}=2 C_{D}^{\prime} / \pi \cosh ^{2} \xi_{0}$ where

$$
C_{D}^{\prime}=e_{z} \cdot\left(\int_{S_{0}} P n \mathrm{~d} A+\frac{2}{R e} \int_{S_{0}} n \times \omega \mathrm{d} A\right),
$$

where $\boldsymbol{n}$ is the unit vector directed inward the ellipsoid while $\boldsymbol{e}_{\boldsymbol{z}}$ denotes the unit vector along the positive $z$-direction; see Landau \& Lifshitz (1987, p. 47).

The drag coefficient will be computed by three different approaches, denoted by $\mathbf{S}$, $P$ and V. These approaches differ in evaluating the drag coefficient due to the pressure. Particular emphasis will be given to the physical significance and numerical implications of each of these approaches.

\subsection{Approach $S$}

This approach has been the most traditional one. The drag coefficient is in terms of the vorticity and its normal derivative on the body surface. Using the spheroidal coordinates, we have

$$
\begin{aligned}
C_{D}=-\frac{8}{3} \tanh \xi_{0} \dot{U}+\frac{4}{R e} \tanh ^{2} \xi_{0} \int_{0}^{\pi}\left(\frac{\partial \omega}{\partial \xi}+\omega \operatorname{coth} \xi\right)_{\xi_{0}} \sin ^{2} \theta \mathrm{d} \theta \\
-\frac{8}{R e} \tanh \xi_{0} \int_{0}^{\pi} \omega_{\xi_{0}} \sin ^{2} \theta \mathrm{d} \theta .
\end{aligned}
$$

The first and second terms on the right-hand side are due to pressure while the remaining term is due to friction. 


\subsection{Approach $P$}

This approach requires evaluating the pressure distribution along each $\theta$. Rewrite equation (2.1) in the form

$$
-\nabla P=\frac{\partial u}{\partial t}+(u \cdot \nabla) u+\frac{2}{R e} \nabla \times \omega .
$$

Taking the $\xi$-component of the equation, we have

$$
\frac{1}{h} \frac{\partial P}{\partial \xi}=-\frac{\partial u_{\xi}}{\partial t}-\frac{1}{2 h} \frac{\partial u^{2}}{\partial \xi}+u_{\theta} \omega-\frac{2}{R e} \frac{1}{h}\left(\frac{\partial \omega}{\partial \theta}+\omega \cot \theta\right) .
$$

Integrating both sides from $\infty$ to a given point by setting the pressure equal to 0 at $\infty$ gives the pressure at that point,

$$
P(\xi, \theta)=\int_{\xi}^{\infty}\left(\frac{\partial u_{\xi}}{\partial t}-\omega u_{\theta}\right) h \mathrm{~d} \xi+\frac{2}{R e} \int_{\xi}^{\infty}\left(\frac{\partial \omega}{\partial \theta}+\omega \cot \theta\right) \mathrm{d} \xi+\frac{1}{2}\left(1-u^{2}\right) .
$$

Specializing to a point on the body surface, we obtain $P_{0}(\theta)$. Substituting the surface pressure $P_{0}$ and the surface vorticity $\omega_{\xi_{0}}$ into $(2.14)$ gives the drag coefficient.

\subsection{Approach V}

The drag coefficient can also be written in terms of a volume integral and a surface integral without involving the derivative of vorticity on the body surface. The following formulae are derived along the lines of Quartapelle \& Napolitano (1983).

Let $\phi=\phi(x)$ be a harmonic function defined in the flow region in $V$, i.e. $\nabla^{2} \phi=0$ in $V$. Furthermore, the function $\phi$ is assumed to decay rapidly away from the ellipsoid. Taking inner products on both sides of $(2.16)$ with $\nabla \phi$ and integrating the resultant expression over the region $V_{R}$ which is bounded by the two ellipsoidal surfaces - the surface of the ellipsoid $S_{0}$ and $S_{R}$ located at $\xi=R$-yields

$$
\begin{aligned}
-\int_{S_{0} \cup S_{R}} P_{n} \cdot \nabla \phi \mathrm{d} A=\int_{S_{0} \cup S_{R}} \phi \frac{\partial u}{\partial t} \cdot n \mathrm{~d} A+\int_{V_{R}}(u \cdot \nabla u) \cdot \nabla \phi \mathrm{d} V \\
+\frac{2}{R e} \int_{S_{0} \cup S_{R}} n \times \omega \cdot \nabla \phi \mathrm{d} A,
\end{aligned}
$$

where we have used the two identities $u \cdot \nabla \phi=\nabla \cdot(u \phi)$ and $(\nabla \times \omega) \cdot \nabla \phi=\nabla \cdot(\omega \times \nabla \phi)$. Recall the identity $u \cdot \nabla u=\frac{1}{2} \nabla u^{2}-u \times \omega$. The second term on the right-hand side of (2.18) can be further rewritten

$$
\int_{V_{R}}(u \cdot \nabla u) \cdot \nabla \phi \mathrm{d} V=\frac{1}{2} \int_{S_{0} \cup S_{R}} u^{2} \nabla \phi \cdot n \mathrm{~d} A-\int_{V_{R}} u \times \omega \cdot \nabla \phi \mathrm{d} V .
$$

Contemplation of (2.14) and (2.18) leads us to define the harmonic function $\phi$ such that it satisfies, on the ellipsoid surface,

$$
\boldsymbol{n} \cdot \boldsymbol{\nabla} \phi=-\boldsymbol{n} \cdot \boldsymbol{e}_{\boldsymbol{z}}
$$

The introduction of $\phi$ enables us to express the contribution from the pressure to the force in terms of the flow quantities $u$ and $\omega$ but not their derivatives. The identities 
(2.18), (2.19) and (2.20) consist of the basic formulae for determining drag in the approach of volume integrals. A harmonic function satisfying the condition in (2.20) is

$$
\begin{gathered}
\phi(\xi, \theta)=C_{1}\left(\cosh \xi \ln \frac{\cosh \xi-1}{\cosh \xi+1}+2\right) \cos \theta, \\
C_{1}=-\left(\ln \frac{\cosh \xi_{0}-1}{\cosh \xi_{0}+1}+2 \frac{\cosh \xi_{0}}{\sinh ^{2} \xi_{0}}\right)^{-1} .
\end{gathered}
$$

This function is bounded and tends to zero rapidly as $\xi \rightarrow \infty$; one, however, still has to be careful in applying it to evaluate the integral involved at the far-field ellipsoidal surface. (For the sphere we have $\phi(r, \theta)=\cos \theta / 2 r^{2}$.)

It is not difficult to see that (2.18) becomes, as $R \rightarrow \infty$,

$$
\begin{aligned}
C_{D P}=\frac{4}{3} C_{2} \dot{U} \tanh \xi_{0} \sinh \xi_{0}-\frac{2}{\pi \cosh ^{2} \xi_{0}} \int_{V} u \times \omega \cdot \nabla \phi \mathrm{d} V \\
\\
\quad+\frac{4}{\pi \operatorname{Re} \cosh ^{2} \xi_{0}} \int_{S_{0}} n \times \omega \cdot \nabla \phi \mathrm{d} A,
\end{aligned}
$$

where $C_{2}=-2 \phi\left(\xi_{0}, 0\right) / \cosh \xi_{0}$. Adding to this result the contribution from friction yields

$$
\begin{aligned}
C_{D}=\frac{4}{3} C_{2} \dot{U} \tanh \xi_{0} \sinh \xi_{0}-\frac{2}{\pi \cosh ^{2} \xi_{0}} \int_{V} u \times \omega \cdot \nabla \phi \mathrm{d} V \\
\quad+\frac{4}{\pi \operatorname{Re} \cosh ^{2} \xi_{0}} \int_{S_{0}} n \times \omega \cdot\left(\nabla \phi+e_{z}\right) \mathrm{d} A .
\end{aligned}
$$

For numerical purposes, we express the result in (2.23) in spheroidal coordinates,

$$
\begin{aligned}
C_{D}= & \frac{4}{3} C_{2} \dot{U} \tanh \xi_{0} \sinh \xi_{0}-\frac{4 C_{1}}{\cosh ^{2} \xi_{0}} \int_{0}^{\pi} \int_{\xi_{0}}^{\infty} \omega\left[\left(\sinh \xi \ln \frac{\cosh \xi-1}{\cosh \xi+1}+\frac{2 \cosh \xi}{\sinh \xi}\right) u_{\theta} \cos \theta\right. \\
& \left.+\left(\cosh \xi \ln \frac{\cosh \xi-1}{\cosh \xi+1}+2\right) u_{\xi} \sin \theta\right] \sinh \xi \sin \theta h \mathrm{~d} \xi \mathrm{d} \theta \\
& -\frac{8}{R e} \tanh \xi_{0}\left(\frac{\phi\left(\xi_{0}, 0\right)}{\cosh \xi_{0}}+1\right) \int_{0}^{\pi} \omega_{\xi_{0}} \sin ^{2} \theta \mathrm{d} \theta
\end{aligned}
$$

\subsection{Numerical implications}

Mathematically, the three approaches are equivalent; however, they may differ substantially from each other in practical numerical implementation. In rapidly changing flows, the vorticity has in general a fairly large gradient near the solid body. The gradient is not obtained directly from the numerical solution but through interpolation; the error inherent in it is inevitably large, therefore, which makes the interpolation not very reliable. Therefore we do not expect approach $S$ to yield accurate results in the early stages of flow development. On the other hand, approaches $P$ and $V$ depend on smoother flow variables, and are much more suitable for evaluating the drag coefficient for rapidly changing flows. Numerical examples will be presented to demonstrate this point. 


\subsection{Physical considerations}

Formula (2.23) can be regarded as a decomposition of the drag coefficient into three parts: one due to acceleration, another to interior (volume) contribution and the third to a surface contribution. That is, we may write the equality: $C_{D}=$ $C_{D A}+C_{D V}+C_{D S}$. Evidently such a decomposition is not unique for the latter two parts; it could, however, be made unique and allow a physically significant explanation provided that we take the viewpoint that the only source of drag, besides the acceleration, is the vorticity rather than its derivative. Notice that the pressure contributes to each of these parts while friction contributes solely to $C_{D S}$. It can be seen from (2.24) that

$$
C_{D S}=\left(\frac{\phi\left(\xi_{0}, 0\right)}{\cosh \xi_{0}}+1\right) C_{D F}
$$

Moreover, in the sense of the present decomposition, the vorticity interior to the flow contributes to $C_{D V}$ according to the volume element

$$
D_{\mathrm{e}}(x) \equiv-2 u \times \omega \cdot \nabla \phi / \pi \cosh ^{2} \xi_{0},
$$

while the surface vorticity contributes to $C_{D S}$ according to $n \times \omega \cdot\left(\nabla \phi+e_{z}\right)$ on the surface of the ellipsoid. The interior element $D_{\mathrm{e}}$ is invariant under the sign reversal of the velocity $\boldsymbol{u}$ while the surface element is not. In other words, the body may experience the same amount of $C_{D V}$ without knowing from which direction the fluid flows. The significance of $D_{\mathrm{e}}(\boldsymbol{x})$ and of $C_{D S}$ will be pursued further in $\S 5$.

\section{Matched asymptotic expansion}

This section is devoted to an asymptotic analysis of $(2.8)$ and (2.10) which is valid at small values of $t$. For small $t$, the flow changes from a potential to a viscous one. It is thus expected that there exists an initial layer within which the flow field varies rapidly with respect to time and space. It proves convenient to develop the solution separately in the viscous wall region (inner region) and an outer region within which the solution is essentially inviscid; the solutions are then matched in an intermediate region. The stream functions identified within these regions are denoted by the superscripts $i$ and o respectively. The stream function and vorticity in the outer region may be represented in the form

$$
\begin{gathered}
\psi=\psi^{\circ}=\psi_{0}^{\circ}(U, \xi, \theta)+\epsilon \psi_{1}^{o}(U, \xi, \theta)+\epsilon^{2} \psi_{2}^{o}(U, \xi, \theta)+\ldots, \\
\omega=\omega^{\circ} \equiv 0,
\end{gathered}
$$

where $\epsilon \equiv(2 t / R e)^{\frac{1}{2}}$. Next we introduce the inner variable $\tilde{\xi} \equiv\left(\xi-\xi_{0}\right) / \epsilon \xi_{0}$ in the inner region. The stream function and vorticity may be represented in the form

$$
\begin{gathered}
\psi=\psi^{\mathrm{i}}=\epsilon \psi_{1}^{\mathrm{i}}(U, \tilde{\xi}, \theta)+\epsilon^{2} \psi_{2}^{\mathrm{i}}(U, \tilde{\xi}, \theta)+\epsilon^{3} \psi_{3}^{\mathrm{i}}(U, \tilde{\xi}, \theta)+\ldots \\
\omega=\omega^{\mathrm{i}}=\epsilon^{-1} \omega_{-1}^{\mathrm{i}}(U, \tilde{\xi}, \theta)+\epsilon^{0} \omega_{0}^{\mathrm{i}}(U, \tilde{\xi}, \theta)+\epsilon \omega_{1}^{\mathrm{i}}(U, \tilde{\xi}, \theta)+\ldots
\end{gathered}
$$

Substituting expansions (3.1) and (3.2) into (2.10) and equating like powers of $\epsilon$ gives

$$
\nabla^{2} \psi_{j}^{o}-2 \frac{\operatorname{coth} \xi}{h^{2}} \frac{\partial \psi_{j}^{o}}{\partial \xi}-2 \frac{\cot \theta}{h^{2}} \frac{\partial \psi_{j}^{0}}{\partial \theta}=0, \quad j=0,1,2, \ldots
$$


For the inner region, substitution of (3.3) and (3.4) into (2.8) and equating like powers of $\epsilon$ yields

$$
\begin{aligned}
& \frac{2}{h_{0}^{2} \xi_{0}^{2}} \frac{\partial^{2} \omega_{-1}^{\mathrm{i}}}{\partial \tilde{\xi}^{2}}+\tilde{\xi} \frac{\partial \omega_{-1}^{\mathrm{i}}}{\partial \tilde{\xi}}+\omega_{-1}^{\mathrm{i}}=0 \\
& \frac{4}{h_{0}^{2} \xi_{0}^{2}} \frac{\partial^{2} \omega_{0}^{\mathrm{i}}}{\partial \tilde{\xi}^{2}}+2 \tilde{\xi} \frac{\partial \omega_{0}^{\mathrm{i}}}{\partial \tilde{\xi}}=-\frac{4}{h_{0}^{2} \xi_{0}} \operatorname{coth} \xi_{0} \frac{\partial \omega_{-1}^{1}}{\partial \tilde{\xi}}+\frac{8 h_{1}}{h_{0}^{2} \xi_{0}} \tilde{\xi} \frac{\partial^{2} \omega_{-1}^{i}}{\partial \tilde{\xi}^{2}} \\
& \frac{4}{h_{0}^{2} \xi_{0}^{2}} \frac{\partial^{2} \omega_{1}^{\mathrm{i}}}{\partial \tilde{\xi}^{2}}+2 \tilde{\xi} \frac{\partial \omega_{1}^{\mathrm{i}}}{\partial \tilde{\xi}}-2 \omega_{1}^{\mathrm{i}}=\frac{2 R e}{h_{0}^{2} \xi_{0} \sinh \xi_{0} \sin \theta} \\
& \times\left(\frac{\partial \psi_{1}^{i}}{\partial \theta} \frac{\partial \omega_{-1}^{i}}{\partial \tilde{\xi}}-\frac{\partial \psi_{1}^{i}}{\partial \tilde{\xi}} \frac{\partial \omega_{-1}^{i}}{\partial \theta}+\cot \theta \omega_{-1}^{i} \frac{\partial \psi_{1}^{i}}{\partial \tilde{\xi}}\right) \\
& -\frac{4}{h_{0}^{2}} \cot \theta \frac{\partial \omega_{-1}^{\mathrm{i}}}{\partial \theta}-\frac{4 \omega_{-1}^{\mathrm{i}}}{\sinh ^{2} \xi_{0} \sin ^{2} \theta}+R e \frac{\partial \omega_{-1}^{\mathrm{i}}}{\partial U} \dot{U} \\
& -\frac{4}{h_{0}^{2} \xi_{0}}\left(\operatorname{coth} \xi_{0} \frac{\partial \omega_{0}^{\mathbf{i}}}{\partial \tilde{\xi}}-\left(\operatorname{cosech}^{2} \xi_{0}+2 h_{1} \operatorname{coth} \xi_{0}\right) \xi_{0} \tilde{\xi} \frac{\partial \omega_{-1}^{\mathrm{i}}}{\partial \tilde{\xi}}\right) \\
& +\frac{8}{h_{0}^{2} \xi_{0}} h_{1} \tilde{\xi} \frac{\partial^{2} \omega_{0}^{1}}{\partial \tilde{\xi}^{2}}-\frac{4}{h_{0}^{2}}\left(h_{1}^{2}-h_{2}\right) \tilde{\xi}^{2} \frac{\partial^{2} \omega_{-1}^{1}}{\partial \tilde{\xi}^{2}}-\frac{4}{h_{0}^{2}} \frac{\partial^{2} \omega_{-1}^{1}}{\partial \theta^{2}} .
\end{aligned}
$$

Similarly, substituting (3.3) and (3.4) into (2.10) gives

$$
\begin{aligned}
-\omega_{-1}^{\mathbf{i}}=\frac{1}{h_{0}^{2} \sinh \xi_{0} \sin \theta} \frac{1}{\xi_{0}^{2}} \frac{\partial^{2} \psi_{1}^{\mathbf{i}}}{\partial \tilde{\xi}^{2}} \\
-\omega_{0}^{\mathrm{i}}=\frac{1}{h_{0}^{2} \sinh \xi_{0} \sin \theta}\left(\frac{1}{\xi_{0}^{2}} \frac{\partial^{2} \psi_{2}^{\mathbf{i}}}{\partial \tilde{\xi}^{2}}-\frac{1}{\xi_{0}} \operatorname{coth} \xi_{0} \frac{\partial \psi_{1}^{\mathrm{i}}}{\partial \tilde{\xi}}-\left(2 h_{1}+\operatorname{coth} \xi_{0}\right) \frac{\tilde{\xi}}{\xi_{0}} \frac{\partial^{2} \psi_{1}^{1}}{\partial \tilde{\xi}^{2}}\right) \\
-\omega_{1}^{1}=\frac{1}{h_{0}^{2} \sinh \xi_{0} \sin \theta}\left\{\frac{1}{\xi_{0}^{2}} \frac{\partial^{2} \psi_{3}^{\mathrm{i}}}{\partial \tilde{\xi}^{2}}+\frac{\partial^{2} \psi_{1}^{\mathrm{i}}}{\partial \theta^{2}}-\frac{1}{\xi_{0}} \operatorname{coth} \xi_{0} \frac{\partial \psi_{2}^{\mathrm{i}}}{\partial \tilde{\xi}}+\operatorname{cosech} \xi_{0} \tilde{\xi} \frac{\partial \psi_{1}^{\mathrm{i}}}{\partial \tilde{\xi}}\right. \\
-\cot \theta \frac{\partial \psi_{1}^{1}}{\partial \theta}-\left(2 h_{1}+\operatorname{coth} \xi_{0}\right) \frac{\tilde{\xi}}{\xi_{0}} \frac{\partial^{2} \psi_{2}^{1}}{\partial \tilde{\xi}^{2}}+\operatorname{coth} \xi_{0}\left(2 h_{1}+\operatorname{coth} \xi_{0}\right) \tilde{\xi} \frac{\partial \psi_{1}^{i}}{\partial \tilde{\xi}} \\
\left.+\left[\left(2 h_{1}+\operatorname{coth} \xi_{0}\right)^{2}-\left(2 h_{1} \operatorname{coth} \xi_{0}+h_{2}+\frac{1}{2}\right)\right] \tilde{\xi}^{2} \frac{\partial^{2} \psi_{1}^{\mathrm{i}}}{\partial \tilde{\xi}^{2}}\right\}
\end{aligned}
$$

where $h_{0}(\theta)=\left(\cosh ^{2} \xi_{0}-\cos ^{2} \theta\right)^{\frac{1}{2}}, h_{1}(\theta)=h_{0}^{-2} \cosh \xi_{0} \sinh \xi_{0}$ and $h_{2}(\theta)=h_{0}^{-2} \cosh 2 \xi_{0}-h_{1}^{2}$. We shall carry out asymptotic expansions only for the first three orders in $\epsilon$. Boundary conditions (2.11) can be expanded in the inner region as

$$
\psi_{j}^{\mathbf{i}}=\frac{\partial \psi_{j}^{1}}{\partial \tilde{\xi}}=0 \quad \text { at } \quad \tilde{\xi}=0, \quad j=1,2, \ldots
$$

Boundary conditions (2.12) imply

$$
\begin{array}{lll}
\psi_{0}^{0}=\frac{1}{2} U \sinh ^{2} \xi \sin ^{2} \theta & \text { as } & \xi \rightarrow \infty, \\
\psi_{j}^{0}=0 & \text { for } j=1,2, \ldots & \text { as } \xi \rightarrow \infty
\end{array}
$$

The leading term in the outer solution which is governed by (3.5) with $j=0$ and boundary condition $(3.9 a)$ was found to be

$$
\psi_{0}^{\circ}=\frac{1}{2} U\left[\sinh ^{2} \xi-C_{1}\left(\sinh ^{2} \xi \ln \frac{\cosh \xi+1}{\cosh \xi-1}-2 \cosh \xi\right)\right] \sin ^{2} \theta
$$


where $C_{1}$ is given by $(2.21 b)$ (cf. Batchelor 1967, p. 457). The solution (3.10) induces through (2.9) a velocity on the surface of the ellipsoid $\xi=\xi_{0}$ :

$$
V_{0}(\theta, t)=-\frac{U \sin \theta}{h_{0}}\left(\phi\left(\xi_{0}, 0\right)+\cosh \xi_{0}\right)
$$

Expand $\psi_{0}^{\circ}$ in terms of the inner variable, as $\xi \rightarrow \xi_{0}$,

$$
\psi_{0}^{0}=\left.\psi_{0}^{o}\right|_{\xi_{0}}-\epsilon h_{0} \xi_{0} \tilde{\xi} V_{0} \sinh \xi_{0} \sin \theta+\left.\frac{1}{2} \epsilon^{2} \xi_{0}^{2} \tilde{\xi}^{2} \frac{\partial^{2} \psi_{0}^{0}}{\partial \xi^{2}}\right|_{\xi_{0}}+\left.\frac{1}{6} \epsilon^{3} \xi_{0}^{3} \tilde{\xi}^{3} \frac{\partial^{3} \psi_{0}^{0}}{\partial \xi^{3}}\right|_{\xi_{0}}+\ldots
$$

where ... denotes higher-order terms. Expansion (3.12) implies that a leading inner solution must be introduced to match with the first non-trivial term on the righthand side of (3.12) in an intermediate region. Notice that $\psi_{0}^{\circ}=0$ at $\xi=\xi_{0}$.

In the inner region, $\psi_{1}^{i}$ and $\omega_{-1}^{i}$ are obtained by solving $(3.6 a)$ and $(3.7 a)$; the obvious boundary conditions are (3.8) for $j=1$ and we further require that $\left.\omega_{-1}^{\mathbf{i}}\right|_{\xi \rightarrow \infty}=0$ and in an intermediate region

$$
\psi_{1}^{i}=-V_{0} h_{0} \xi_{0} \tilde{\xi} \sinh \xi_{0} \sin \theta+\ldots
$$

For simplicity, throughout this section we will not use explicitly the intermediate variable; any intermediate matching and order analysis can be made clear by introducing the variable $\zeta=\epsilon^{\frac{1}{a}} \tilde{\xi}$.

Again, for simplicity we define $\eta=\frac{1}{2} \xi_{0} h_{0} \tilde{\xi}$. Writing $\psi_{1}^{1}=-V_{0} \sinh \xi_{0} \sin \theta f_{1}(\eta)$, we have from $(3.6 a)$ and $(3.7 a)$ by eliminating $\omega_{-1}^{\mathbf{i}}$

$$
\begin{gathered}
f_{1}^{\mathrm{iv}}+2 \eta f_{1}^{\prime \prime \prime}+2 f_{1}^{\prime \prime}=0, \\
f_{1}=f_{1}^{\prime}=0 \quad \text { at } \quad \eta=0, \\
f_{1}^{\prime}(\eta)=2, \quad f_{1}^{\prime \prime}(\eta)=0 \quad \text { as } \eta \rightarrow \infty
\end{gathered}
$$

where a prime denotes differentiation once with respect to $\eta$. The solution to (3.13ac) is the classical solution obtained by Blasius (1908),

$$
f_{1}=2 \eta \operatorname{erf} \eta+2 \pi^{\frac{1}{2}}\left(\mathrm{e}^{-\eta^{2}}-1\right) .
$$

Thus we have $\quad \psi_{1}^{\mathrm{i}}=-2 V_{0} \sin \theta \sinh \xi_{0}\left[\eta \operatorname{erf} \eta+\pi^{-\frac{1}{2}}\left(\mathrm{e}^{-\eta^{2}}-1\right)\right]$.

The corresponding vorticity is given by

$$
\omega_{-1}^{i}=V_{0} \pi^{-\frac{1}{2}} \mathrm{e}^{-\eta^{2}}
$$

It follows from (3.15) that, as $\eta \rightarrow \infty$,

$$
\epsilon \psi_{1}^{\mathrm{i}}=-\epsilon V_{0} h_{0} \xi_{0} \tilde{\xi} \sinh \xi_{0} \sin \theta+\epsilon 2 V_{0} \pi^{-\frac{1}{2}} \sinh \xi_{0} \sin \theta
$$

Contemplation of (3.12) and (3.17) leads us to introduce the second term $\epsilon \psi_{1}^{\circ}$ in the outer solution so that $\psi_{0}^{\mathrm{o}}+\epsilon \psi_{1}^{\mathrm{o}}$ matches with $\epsilon \psi_{1}^{\mathrm{i}}$ in an intermediate region; this leads to

with

$$
\nabla^{2} \psi_{1}^{o}-2 \frac{\operatorname{coth} \xi}{h^{2}} \frac{\partial \psi_{1}^{\circ}}{\partial \xi}-2 \frac{\cot \theta}{h^{2}} \frac{\partial \psi_{1}^{\circ}}{\partial \theta}=0
$$

$$
\psi_{1}^{o}\left(\xi=\xi_{0}, \theta, t\right)=2 V_{0} \pi^{-\frac{1}{2}} \sinh \xi_{0} \sin \theta
$$

We do not find a closed-form solution to $(3.18 a-c)$, which has to be solved numerically. Notice that $\epsilon \psi_{1}^{\circ}$ induces a slip velocity $\epsilon V_{1}$ on $\xi=\xi_{0}$ which is given by

$$
V_{1}=\left.\frac{-1}{h \sinh \xi \sin \theta} \frac{\partial \psi_{1}^{\circ}}{\partial \xi}\right|_{\xi-\xi_{0}} .
$$


Expand $\epsilon \psi_{1}^{\circ}$ in terms of the inner variable $\tilde{\xi}$, as $\xi \rightarrow \xi_{0}$,

$$
\epsilon \psi_{1}^{\mathrm{o}}(\xi, \theta, t)=\epsilon 2 V_{0} \pi^{-\frac{1}{2}} \sinh \xi_{0} \sin \theta-\epsilon^{2} V_{1} h_{0} \xi_{0} \tilde{\xi} \sinh \xi_{0} \sin \theta+\left.\frac{1}{2} \epsilon^{3} \xi_{0}^{2} \tilde{\xi}^{2} \frac{\partial^{2} \psi_{1}^{\mathrm{o}}}{\partial \xi^{2}}\right|_{\xi-\xi_{0}}+\ldots
$$

We have now obtained $\psi_{0}^{o}, \psi_{1}^{o}$ and $\psi_{1}^{\mathrm{i}}$. Before proceeding to higher-order matching, we observe from (3.12), (3.17) and (3.20) that the equality

$$
\begin{array}{r}
\psi_{0}^{0}+\epsilon \psi_{1}^{0}-\epsilon \psi_{1}^{\mathrm{i}}=\left.\frac{1}{2} \epsilon^{2} \xi_{0}^{2} \tilde{\xi}^{2} \frac{\partial^{2} \psi_{0}^{0}}{\partial \xi^{2}}\right|_{\xi_{0}}-\epsilon^{2} V_{1} h_{0} \xi_{0} \tilde{\xi} \sinh \xi_{0} \sin \theta+\left.\frac{1}{6} \epsilon^{3} \xi_{0}^{3} \tilde{\xi}^{3} \frac{\partial^{3} \psi_{0}^{0}}{\partial \xi^{3}}\right|_{\xi_{0}} \\
+\left.\frac{1}{2} \epsilon^{3} \xi_{0}^{2} \tilde{\xi}^{2} \frac{\partial^{2} \psi_{1}^{0}}{\partial \xi^{2}}\right|_{\xi_{0}}+\ldots
\end{array}
$$

holds in an intermediate region. Therefore, at the next order of matching, $\psi_{2}^{i}$ has to be introduced so that in the intermediate region

$$
\psi_{2}^{\mathrm{i}}(\tilde{\xi}, \theta, t)=\left.\frac{1}{2} \xi_{0}^{2} \tilde{\xi}^{2} \frac{\partial^{2} \psi_{0}^{\circ}}{\partial \xi^{2}}\right|_{\xi_{0}}-V_{1} h_{0} \xi_{0} \tilde{\xi} \sinh \xi_{0} \sin \theta+\ldots
$$

Employing (3.5) for $j=0$ at $\xi=\xi_{0}$, we find

$$
\left.\frac{\partial^{2} \psi_{0}^{\mathrm{o}}}{\partial \xi^{2}}\right|_{\xi_{0}}=-V_{0} h_{0} \operatorname{coth} \xi_{0} \sinh \xi_{0} \sin \theta
$$

A careful consideration of $(3.6 b),(3.7 b),(3.21)$ and $(3.23)$ leads us to assume $\psi_{2}^{i}$ to be of the form

$$
\psi_{2}^{\mathrm{i}}=\left(-V_{1} f_{20}(\eta)-8 V_{0} \frac{h_{1}}{h_{0}} f_{21}(\eta)-2 V_{0} \frac{\operatorname{coth} \xi_{0}}{h_{0}} f_{22}(\eta)\right) \sinh \xi_{0} \sin \theta
$$

The first term on the right-hand side of (3.24) is introduced to fulfil the matching condition (3.22) while the other two terms are to take care of the inhomogeneous terms on the right-hand side of (3.6b). Substituting (3.24) into (3.7b) and eliminating $\omega_{0}^{1}$ through the use of $(3.6 b)$ yields

$$
\begin{gathered}
f_{20}^{\mathrm{iv}}+2 \eta f_{20}^{\prime \prime \prime}=0, \quad f_{20}^{\prime}(0)=f_{20}(0)=0, \\
f_{20}^{\prime}(\eta) \rightarrow 2, \quad f_{20}^{\prime \prime}(\eta) \rightarrow 0 \text { as } \eta \rightarrow \infty ; \\
f_{21}^{\mathrm{iv}}+2 \eta f_{21}^{\prime \prime \prime}=\eta f_{1}^{\prime \prime \prime}+\left(\eta^{2}+1\right) f_{1}^{\prime \prime \prime}+\eta f_{1}^{\prime \prime}, \\
f_{21}^{\prime}(0)=f_{21}(0)=0, \\
f_{21}^{\prime}(\eta) \rightarrow 0, \quad f_{21}^{\prime \prime}(\eta) \rightarrow 0, \quad \text { as } \eta \rightarrow \infty ; \\
f_{22}^{\mathrm{iv}}+2 \eta f_{22}^{\prime \prime \prime}=2 f_{1}^{\prime \prime \prime}+2 \eta f_{1}^{\prime \prime}, \quad f_{22}(0)=f_{22}^{\prime}(0)=0, \\
f_{22}^{\prime}(\eta) \rightarrow 2 \eta, \quad f_{22}^{\prime \prime}(\eta) \rightarrow 2 \quad \text { as } \eta \rightarrow \infty .
\end{gathered}
$$

The solutions to (3.25), (3.26) and (3.27) are given by

$$
\begin{gathered}
f_{20}=2 \eta+\pi^{\frac{1}{2}} \eta^{2} \operatorname{erfc} \eta-\eta \mathrm{e}^{-\eta^{2}}-\frac{1}{2} \pi^{\frac{1}{2}} \operatorname{erf} \eta, \\
f_{21}=-\frac{1}{8} \eta^{2} \operatorname{erfc} \eta+\frac{1}{16} \operatorname{erf} \eta-\frac{1}{8} \eta \mathrm{e}^{-\eta^{2}}, \\
f_{22}=\frac{1}{2} \eta^{2}+\frac{1}{2} \eta^{2} \operatorname{erf} \eta+\frac{1}{2} \pi^{-\frac{1}{2}} \eta \mathrm{e}^{-\eta^{2}}-\frac{1}{4} \operatorname{erf} \eta .
\end{gathered}
$$

The corresponding vorticity $\omega_{0}^{\mathbf{i}}$ is given by $\omega_{0}^{1}=V_{1} \frac{1}{2} \pi^{\frac{1}{2}} \operatorname{erfc} \eta-V_{0} \frac{\operatorname{coth} \xi_{0}}{2 h_{0}}\left(\operatorname{erfc} \eta+2 \pi^{-\frac{1}{2}} \eta \mathrm{e}^{-\eta^{2}}\right)-V_{0} \frac{h_{1}}{h_{0}}\left(\frac{1}{2} \operatorname{erfc} \eta+\pi^{-\frac{1}{2}} \eta \mathrm{e}^{-\eta^{2}}+2 \pi^{-\frac{1}{2}} \eta^{3} \mathrm{e}^{-\eta^{2}}\right)$. 
For further matching we observe from (3.24) and (3.28)-(3.30) that, as $\eta \rightarrow \infty$,

$$
\begin{aligned}
\epsilon^{2} \psi_{2}^{\mathrm{i}}(\tilde{\xi}, \theta, t)=\frac{1}{2} \epsilon^{2} \xi_{0}^{2} & \left.\tilde{\xi}^{2} \frac{\partial^{2} \psi_{0}^{\circ}}{\partial \xi^{2}}\right|_{\xi_{0}}-\epsilon^{2} V_{1} h_{0} \xi_{0} \tilde{\xi} \sinh \xi_{0} \sin \theta \\
& +\epsilon^{2}\left(\frac{1}{2} \pi^{\frac{1}{2}} V_{1}-\frac{1}{2} \frac{h_{1}}{h_{0}} V_{0}+\frac{1}{2 h_{0}} V_{0} \operatorname{coth} \xi_{0}\right) \sinh \xi_{0} \sin \theta
\end{aligned}
$$

Subtracting (3.32) from (3.21) reveals that the next term $\psi_{2}^{0}$ in the outer solution has to fulfil the conditions

$$
\begin{gathered}
\psi_{2}^{\circ}\left(\xi_{0}, \theta, t\right)=\left(\frac{1}{2} \pi^{\frac{1}{2}} V_{1}-\frac{1}{2} \frac{h_{1}}{h_{0}} V_{0}+\frac{1}{2 h_{0}} V_{0} \operatorname{coth} \xi_{0}\right) \sinh \xi_{0} \sin \theta \\
\psi_{2}^{\mathrm{o}}(\xi=\infty)=0
\end{gathered}
$$

Again, (3.5) for $j=2$ with these conditions has to be solved numerically. Just like $\psi_{0}^{\circ}$ and $\psi_{1}^{\circ}, \psi_{2}^{o}$ induces a slip velocity on $\xi=\xi_{0}$,

$$
V_{2}=\left.\frac{-1}{h \sinh \xi_{0} \sin \theta} \frac{\partial \psi_{2}^{\circ}}{\partial \xi}\right|_{\xi_{0}}
$$

Our final matching will be made with $\psi_{3}^{1}$. First we expand, as $\xi \rightarrow \xi_{0}$,

$$
\begin{aligned}
\epsilon^{2} \psi_{2}^{\mathrm{o}}(\xi, \theta, t)=\epsilon^{2}\left(\frac{1}{2} \pi^{\frac{1}{2}} V_{1}-\frac{h_{1}}{2 h_{0}} V_{0}\right. & \left.+\frac{1}{2 h_{0}} V_{0} \operatorname{coth} \xi_{0}\right) \\
& \sinh \xi_{0} \sin \theta \\
& -\epsilon^{3} V_{2} h_{0} \tilde{\xi}_{\sinh } \xi_{0} \sin \theta+\ldots
\end{aligned}
$$

Combining (3.21), (3.32) and (3.36), we have

$$
\begin{array}{r}
\psi_{0}^{\mathrm{o}}+\epsilon \psi_{1}^{\mathrm{o}}+\epsilon^{2} \psi_{2}^{\mathrm{o}}-\epsilon \psi_{1}^{\mathrm{i}}-\epsilon^{2} \psi_{2}^{\mathrm{i}}=-\epsilon^{3} V_{2} h_{0} \tilde{\xi} \sinh \xi_{0} \sin \theta+\left.\frac{1}{6} \epsilon^{3} \xi_{0}^{3} \tilde{\xi}^{3} \frac{\partial^{3} \psi_{0}^{\mathrm{o}}}{\partial \xi^{3}}\right|_{\xi_{0}} \\
+\left.\frac{1}{2} \epsilon^{3} \xi_{0}^{2} \tilde{\xi}^{2} \frac{\partial^{2} \psi_{1}^{\mathrm{o}}}{\partial \xi^{2}}\right|_{\xi_{0}}+\ldots
\end{array}
$$

which holds in an intermediate region. Therefore, at the next order of matching, $\psi_{3}^{i}$ must be introduced such that in the intermediate region

$$
\psi_{3}^{\mathrm{i}}(\tilde{\xi}, \theta, t)=-V_{2} h_{0} \tilde{\xi} \sinh \xi_{0} \sin \theta+\left.\frac{1}{6} \xi_{0}^{3} \tilde{\xi^{3}} \frac{\partial^{3} \psi_{0}^{0}}{\partial \xi^{3}}\right|_{\xi_{0}}+\left.\frac{1}{2} \xi_{0}^{2} \tilde{\xi}^{2} \frac{\partial^{2} \psi_{1}^{0}}{\partial \xi^{2}}\right|_{\xi_{0}}+\ldots
$$

Employing (3.5) for $j=1,0$ at $\xi=\xi_{0}$ we have respectively,

and

$$
\left.\frac{\partial^{2} \psi_{1}^{o}}{\partial \xi^{2}}\right|_{\xi_{0}}=-V_{1} h_{0} \cosh \xi_{0} \sin \theta
$$

$$
\begin{aligned}
\left.\frac{\partial^{3} \psi_{0}^{o}}{\partial \xi^{3}}\right|_{\xi_{0}}=\left(V_{0, \theta \theta} h_{0}\right. & \sin \theta+2 V_{0, \theta} h_{0, \theta} \sin \theta+V_{0} h_{0, \theta \theta} \sin \theta \\
& \left.+V_{0} h_{0, \theta} \cos \theta+V_{0, \theta} h_{0} \cos \theta-\frac{V_{0} h_{0}}{\sin \theta}-V_{0} h_{0} \sin \theta\right) \sinh \xi_{0}
\end{aligned}
$$


where a subscript $\theta$ denotes differentiation once with respect to $\theta$. Recall $\omega_{0}^{i}$ in (3.31). It is sophisticated enough to investigate the solution form of $\psi_{0}^{\mathrm{i}}$ through (3.6c), (3.7c) and (3.38)-(3.40). Nevertheless, by eliminating $\omega_{1}^{\mathbf{i}}$ in $(3.7 c)$ through the use of $(3.6 c)$, we find it adequate to assume $\psi_{3}^{i}$ to be of the form

$$
\begin{aligned}
\psi_{3}^{1}= & -V_{2} \sinh \xi_{0} \sin \theta f_{30}(\eta)-4 V_{1} \frac{h_{1}}{h_{0}} \sinh \xi_{0} \sin \theta f_{31}(\eta) \\
& -2 V_{1} \frac{1}{h_{0}} \cosh \xi_{0} \sin \theta f_{32}(\eta)+4 V_{0} \frac{1}{h_{0}} \operatorname{cosech} \xi_{0} \sin \theta f_{33}(\eta) \\
& -4 V_{0} \frac{1}{h_{0}} \operatorname{coth} \xi_{0} \cosh \xi_{0} \sin \theta f_{34}(\eta)+4 V_{0} \frac{h_{1}}{h_{0}} \cosh \xi_{0} \sin \theta f_{35}(\eta) \\
& +4 V_{0} \frac{h_{1}^{2}}{h_{0}} \sinh \xi_{0} \sin \theta f_{36}(\eta)+4 V_{0} \frac{h_{1}^{2}}{h_{0}^{2}} \sinh \xi_{0} \sin \theta f_{37}(\eta) \\
& +4 V_{0} \frac{4 h_{1}+\cosh \xi_{0}}{h_{0}^{2}} \cosh \xi_{0} \sinh \xi_{0} \sin \theta f_{38}(\eta) \\
& +4 V_{0} \frac{h_{1}}{h_{0}^{2}} \cosh \xi_{0} \sin \theta f_{39}(\eta)+4 V_{0} \frac{h_{2}}{h_{0}^{2}} \sinh \xi_{0} \sin \theta f_{310}(\eta) \\
& +2 V_{0} \frac{1}{h_{0}^{2}} \sinh \xi_{0} \sin \theta f_{311}(\eta)+4 V_{0} \frac{1}{h_{0}^{2}} \operatorname{coth} \xi_{0} \sinh \xi_{0} \sin \theta f_{312}(\eta) \\
& +\frac{4 V_{0}}{\sinh \xi_{0} \sin \theta} f_{313}(\eta)-\frac{\partial V_{0}}{\partial U} \dot{U} R e \sinh \xi_{0} \sin \theta f_{314}(\eta) \\
& +\frac{R e V_{0}^{2}}{h_{0}} \sinh \xi_{0} \cos \theta f_{320}(\eta)+R e \frac{V_{0}^{2}}{h_{0}^{2}} h_{0, \theta} \sinh \xi_{0} \sin \theta f_{321}(\eta) \\
& +\frac{4 V_{0}}{h_{0}^{2} \sin \theta} \sinh \xi_{0} f_{315}(\eta)-\frac{4}{h_{0}^{2}} \sinh \xi_{0}\left[\sin \theta V_{0, \theta \theta}+\cot \theta V_{0, \theta}\right] f_{316}(\eta) \\
& +\frac{4}{h_{0}^{3}} \sinh \xi_{0}\left[2 h_{0, \theta} V_{0, \theta} \sin \theta+h_{0, \theta} V_{0} \cos \theta+h_{0, \theta \theta} V_{0} \sin \theta\right] f_{317}(\eta) \\
& +4 V_{0} \frac{h_{0, \theta}^{2}}{h_{0}^{4}} \sinh \xi_{0} \sin \theta f_{318}(\eta)-R e \frac{V_{0, \theta} V_{0}}{h_{0}} \sinh \xi_{0} \sin \theta f_{319}(\eta) \\
& =1(\eta) \\
&
\end{aligned}
$$

Finding the full solution is quite complicated; we are only interested in those terms that contain the Reynolds number, which may contribute to the nonlinear modification of the leading behaviour. Some manipulations yield the results

$$
\begin{aligned}
f_{314}= & -\frac{2}{3} \eta^{3} \operatorname{erfc} \eta-\frac{1}{3} \pi^{-\frac{1}{2}}\left(\mathrm{e}^{-\eta^{2}}-1\right)+\frac{2}{3} \pi^{-\frac{1}{2}} \eta^{2} \mathrm{e}^{-\eta^{2}}, \\
f_{319}= & \frac{11}{6} \pi^{-\frac{1}{2}} \mathrm{e}^{-\eta^{2}} \operatorname{erfc} \eta-\frac{8}{3}(2 \pi)^{-\frac{1}{2}} \operatorname{erfc}(\sqrt{ } 2 \eta)+\frac{1}{3} \eta^{3} \operatorname{erfc}^{2} \eta-\frac{2}{3} \pi^{-\frac{1}{2}} \eta^{2} \mathrm{e}^{-\eta^{2}} \operatorname{erfc} \eta \\
& +\frac{1}{3} \pi^{-1} \eta \mathrm{e}^{-2 \eta^{2}}-\frac{1}{2} \eta \operatorname{erfc}^{2} \eta+\pi^{-\frac{1}{2}}\left(\frac{4}{9} \pi^{-1}-\frac{3}{2}\right) \mathrm{e}^{-\eta^{2}}-\left(1+\frac{4}{9} \pi^{-1}\right) \eta^{3} \operatorname{erfc} \eta \\
& +\pi^{-\frac{1}{2}}\left(1+\frac{4}{9} \pi^{-1}\right) \eta^{2} \mathrm{e}^{-\eta^{2}}+\frac{2}{3} \pi^{-\frac{1}{2}} \operatorname{erfc} \eta+\left(\frac{1}{2}-\frac{2}{3} \pi^{-1}\right) \eta \operatorname{erfc} \eta+\pi^{-\frac{1}{2}}\left(\frac{4}{3} \sqrt{ } 2-\frac{4}{9} \pi^{-1}-1\right), \\
f_{320}= & -\frac{1}{3} \eta^{3} \operatorname{erf} \eta \operatorname{erfc} \eta-\frac{8}{9} \pi^{-1} \eta^{3} \operatorname{erfc} \eta-\frac{1}{3} \pi^{-\frac{1}{2}} \eta^{2} \mathrm{e}^{-\eta^{2}}(1-2 \operatorname{erf} \eta) \\
& +\frac{8}{9} \pi^{-\frac{3}{2}} \eta^{2} \mathrm{e}^{-\eta^{2}}-\frac{4}{3} \pi^{-1} \eta(1-2 \operatorname{erf} \eta)+\frac{1}{2} \eta \operatorname{erf} \eta(2-\operatorname{erf} \eta)+\frac{1}{3} \pi^{-1} \eta \mathrm{e}^{-2} \eta^{2} \\
& +\frac{7}{6} \pi^{-\frac{1}{2}} \mathrm{e}^{-\eta^{2}} \operatorname{erf} \eta-\frac{1}{3} \pi^{-\frac{1}{2}}\left(1-\frac{8}{3} \pi^{-1}\right)\left(\mathrm{e}^{-\eta^{2}}-1\right)-\frac{2}{3} \sqrt{ } 2 \pi^{-\frac{1}{2}} \operatorname{erf}(\sqrt{ } 2 \eta)+\frac{2}{3} \pi^{-\frac{1}{2}} \operatorname{erf} \eta, \\
f_{321}= & \frac{1}{2}\left(f_{320}-f_{319}\right) .
\end{aligned}
$$


The corresponding vorticity $\omega_{1}^{i}$ can be obtained according to $(3.7 c)$; the details will not be given here. This completes our derivation of the asymptotic expansion.

Some information concerning the initial drag coefficient is now readily available from the asymptotic result. Recall that the first two terms on the right-hand side of (2.15) contribute to $C_{D P}$ while the last term is $C_{D F}$. If the ellipsoid is abruptly started from rest to a constant speed, i.e. $\dot{U}=0$ for $t>0$, the leading terms of the total drag coefficient are then given by $C_{D}=C_{D F}+C_{D P}$, where

$$
C_{D F}=-8 \frac{\tanh \xi_{0}}{(2 \pi t R e)^{\frac{1}{2}}} \int_{0}^{\pi} V_{0} \sin ^{2} \theta \mathrm{d} \theta
$$

and

$$
C_{D P}=-4 \frac{\tanh ^{2} \xi_{0}}{(2 \pi t R e)^{\frac{1}{2}}} \int_{0}^{\pi}\left(2 V_{1} h_{0}-\frac{\operatorname{coth} \xi_{0}}{2 \pi^{\frac{1}{2}}} V_{0}\right) \sin ^{2} \theta \mathrm{d} \theta
$$

Finally, it has to be remarked that the asymptotic result is valid only within an initial portion of time since the analysis assumes (tacitly) that the nonlinear effects do not enter the analysis until the third order (cf. (3.6)). Order analysis shows that this assumption can only be valid if $\epsilon R e \ll 1$, which implies that $t \ll 1 /(2 R e)$ or at best $t=O(1 / R e)$; these conditions are much more restricted than $\epsilon \ll 1$. The literature appears to indicate that the validity of such an expansion is not so limited; most authors have used their asymptotic results up to $t=1$ or even longer. In the present study, we carry out the asymptotic expansion and the numerical solution separately; the results are then compared to each other to ascertain the time interval of validity for the asymptotic expansion and the resolvability of the hybrid vortex method for initial flows.

\section{Hybrid vortex method}

The present vortex method can be abbreviated as follows. The vorticity field is approximated by a sum of 'blob' functions, called (computational) vortex blobs or simply vortices. Each vortex evolves in a Lagrangian manner, carrying with itself a circulation determined from the vorticity. The vorticity is obtained by solving the vorticity diffusion equation on a grid by finite differences. The circulations are then redistributed and converted back to the vorticity on the grid, which is thus updated. Below, the hybrid vortex method will only be described for the ellipsoid; the method for the sphere is in the same spirit except that spherical coordinates are adopted.

At the beginning of each step there are only vortices centred at mesh points. Therefore, first, we approximate the vorticity field by

$$
\omega(t, x)=\sum_{j=1}^{N} \Gamma_{j} f_{j}\left(x-x_{j}\right)
$$

where $N$ denotes the number of vortices (or mesh points) and $f_{j}(x)$ is the blob function. $\Gamma_{j}$ and $x_{j}$ are respectively the circulation and position associated with the $j$ th vortex blob. In practice, the function $f_{j}(x)$ is conveniently taken to be an indicator function so that the vorticity is constant over the given indicator set, outside which the blob function is zero. Highly accurate blob functions may be available in the form of combinations of some elementary functions; see, for example, Hald (1979) and Beale \& Majda (1985).

Next we describe the polar coordinates $\left(\xi^{\prime}, \theta\right)$ for the solution of $(2.10)$ and for the creation of vorticity along the ellipsoid surface. The coordinate system is also used 
to solve the vorticity diffusion equation in order to take into account the effect of viscous diffusion. In terms of $\left(\xi^{\prime}, \theta\right)$ the $(2.10)$ may be written

$$
\frac{\partial^{2} \psi}{\partial \theta^{2}}-\cot \theta \frac{\partial \psi}{\partial \theta}+a\left(\xi^{\prime}\right) \frac{\partial^{2} \psi}{\partial \xi^{\prime 2}}+b\left(\xi^{\prime}\right) \frac{\partial \psi}{\partial \xi^{\prime}}=-\omega h^{2} \sinh \xi \sin \theta
$$

where

$$
\begin{gathered}
\xi \equiv \xi_{0}+A \xi^{\prime}+B \xi^{\prime 2}, \quad a\left(\xi^{\prime}\right)=\left(\mathrm{d} \xi^{\prime} / \mathrm{d} \xi\right)^{2}=\left(A+2 B \xi^{\prime}\right)^{-2}, \\
b\left(\xi^{\prime}\right)=\frac{\mathrm{d}^{2} \xi^{\prime}}{\mathrm{d} \xi^{2}}-\operatorname{coth} \xi \frac{\mathrm{d} \xi^{\prime}}{\mathrm{d} \xi}=-2 B\left(A+2 B \xi^{\prime}\right)^{-3}-\operatorname{coth} \xi\left(A+2 B \xi^{\prime}\right)^{-1} .
\end{gathered}
$$

For each time increment, (4.2) is solved on a mesh with a uniform mesh size in the coordinate system $\left(\xi^{\prime}, \theta\right)$, defined over an annular region outside the ellipsoid surface $\left(1<\xi<\xi_{R}\right)$. Fine resolution is required near the surface of the ellipsoid. The constants $A, B$ in (4.3a) are fixed by the radial mesh spacing at the ellipsoid surface, and by the value of the outer surface at $\xi_{R}$. The value of $\xi_{R}$ must be sufficiently large for (4.1) to be an adequate approximation and for all the vortices to be contained within the mesh.

Denote by $(i, j)$ the node of the mesh; $\xi^{\prime}=j$ and $\theta=i \Delta \theta(\Delta \theta=\pi / p, 0 \leqslant j \leqslant q-1)$. Let $\psi(i, j)$ be the nodal value of the stream function at $(i, j)$. Equation $(4.2)$ is solved by the method of operator splitting (ADI); that is, to find the steady-state solution of the 'pseudo' diffusion equation:

$$
\frac{\partial \psi}{\partial \tau}=\alpha\left[\frac{1}{h^{2} \sinh \xi \sin \theta}\left(\frac{\partial^{2} \psi}{\partial \theta^{2}}-\cot \theta \frac{\partial \psi}{\partial \theta}+a\left(\xi^{\prime}\right) \frac{\partial^{2} \psi}{\partial \xi^{\prime 2}}+b\left(\xi^{\prime}\right) \frac{\partial \psi}{\partial \xi^{\prime}}\right)+\omega\right],
$$

where $\alpha$ is a positive constant and $\tau$ is called the pseudo-time. Thus, a complete iteration cycle would consist of a sweep over all rows followed by a sweep over the column. This yields a set of $p$ tridiagonal equations in $\xi^{\prime}$ and a set of $q$ tridiagonal equations in $\theta$, which can each be solved efficiently by Gaussian elimination. Once (4.2) is solved, the stream function is used to update the vorticity on the ellipsoid surface so that the no-slip condition is satisfied. Notice that along the ellipsoid surface, (4.2) reduces to

$$
\left.\frac{a\left(\xi^{\prime}\right)}{h^{2} \sinh \xi \sin \theta} \frac{\partial^{2} \psi}{\partial \xi^{\prime 2}}\right|_{\xi^{\prime}=0}=-\omega(i, 0)
$$

Imagine that the stream function can be extended across the ellipsoid surface to possess meaningful values $\psi(i,-1)$. Then, at least to a first-order approximation, we require $\psi(i,-1)=\psi(i, 1)$ so that the tangential velocity along the ellipsoid surface is zero. Applying the central finite difference to (4.5) then yields the surface vorticity

$$
\omega(i, 0)=-\frac{2 a(0) \psi(i, 1)}{h^{2} \sinh \xi \sin \theta}
$$

The value of $\omega(i, 0)$ in (4.5) can be evaluated accurately by interpolating the stream function using three or more radial mesh values near to the surface of the ellipsoid, followed by differentiation. With the solution of $(4.2)$ and the surface vorticity $\omega(i$, 0 ) in (4.6), the next step is to solve the diffusion equation on the grid by a finitedifference method,

$$
\frac{\partial \omega}{\partial t}=\frac{2}{R e}\left[\frac{1}{h^{2}}\left(\frac{\partial^{2} \omega}{\partial \theta^{2}}+\cot \theta \frac{\partial \omega}{\partial \theta}+a\left(\xi^{\prime}\right) \frac{\partial^{2} \omega}{\partial \xi^{\prime 2}}+c\left(\xi^{\prime}\right) \frac{\partial \omega}{\partial \xi^{\prime}}\right)-\frac{\omega}{\sinh ^{2} \xi \sin ^{2} \theta}\right],
$$


where $\quad c\left(\xi^{\prime}\right)=\frac{\mathrm{d}^{2} \xi^{\prime}}{\mathrm{d} \xi^{2}}+\operatorname{coth} \xi \frac{\mathrm{d} \xi^{\prime}}{\mathrm{d} \xi}=-2 B\left(A+2 B \xi^{\prime}\right)^{-3}+\operatorname{coth} \xi\left(A+2 B \xi^{\prime}\right)^{-1}$

From the solution we construct the circulations to be convected by the vortices on the grid. Let $A_{i j}$ be the cell determined by $\left(i-\frac{1}{2}, j\right),\left(i+\frac{1}{2}, j\right),\left(i, j-\frac{1}{2}\right)$ and $\left(i, j+\frac{1}{2}\right)$. Each $A_{i j}$ corresponds to an indicator set in the original $\boldsymbol{x}$-space. The circulation associated with the vortex at $(i, j)$ is then determined approximately by

$$
\Gamma(i, j)=\left.\omega(i, j) h^{2} \Delta \theta \frac{\mathrm{d} \xi}{\mathrm{d} \xi^{\prime}}\right|_{\xi^{\prime}=j} .
$$

The formula is obtained by applying the midpoint rule for evaluating, in the $\left(\xi^{\prime}, \theta\right)$ plane, the integral of the vorticity over the area corresponding to $A_{i j}$. According to the Helmholtz theorem the strength of a vortex is invariant under Euler's equation of motion; the vortex carrying $\Gamma(i, j)$ is convected to a new location with the velocity determined from the formula (2.9). The circulation $\Gamma(i, j)$ is then redistributed between the four corner nodes of the cell in which the vortex is contained according to the area-weighting scheme suggested by Christiansen (1973). The redistribution of circulations completes a cycle of computation; recall that the conversion of circulation into vorticity, and vice versa, is made through the formula (4.8). The last step updates the vorticity on the grid. The present vortex method therefore, for each time increment, consists of the following solution steps :

(i) equation (2.5) for the stream function is solved on the grid; the solution is used to update the vorticity along the ellipsoid surface to satisfy the no-slip condition;

(ii) equation (4.7) is solved on the grid by a finite difference; the circulation associated with each vortex on the grid is then evaluated according to the formula (4.8);

(iii) each vortex on the grid together with the associated circulation is convected with the velocity determined by (2.9) using the values of the stream function obtained in (i);

(iv) redistribute all the circulations; add at each mesh point the circulations contributed and convert the result into vorticity, again, according to the formula (4.8).

Notice that whenever the circulation is convected and redistributed, both the effects of vortex convection and stretching are automatically taken into consideration. The method described above is obviously of fractional step type. Some evidence of the validity of the viscous splitting method for the Navier-Stokes equations can be found in the article by Beale \& Majda (1981). Time accuracy can be improved by adopting a midpoint rule for evaluating the velocities of the vortices on the grid. One way of achieving this is to calculate the velocities using the average of the current vorticity and a predicted vorticity (both on the grid) at the next time step. This modified procedure is conceived to have second-order accuracy in time, and is actually used in computation. In the present approach vortices on the surface of the ellipsoid are not convected into the flow, while the diffusion of vorticity is achieved through solving the diffusion equation. The method is therefore completely deterministic, and is fundamentally different from those using random walks to simulate the effect of viscous diffusion. The manner in which the vortices are convected and the circulations are redistributed consist exactly of that of the vortexin-cell method for inviscid flow. It is further noticed that the area-weighting scheme with the vortex-in-cell method, which locally smooths the vorticity field, is a mild 
source of numerical diffusion though its effect becomes smaller as the mesh becomes finer.

\section{Numerical results and discussions}

Axisymmetric flow around an ellipsoid could depend significantly on the Reynolds number and the geometry of the ellipsoid. In the present study, we consider the limiting case of a sphere and an ellipsoid of axis ratio $2: 1$. The flow is started impulsively from rest. The Reynolds number based on the diameter, i.e. $R e_{d}$, varies between 100 and 3000 or even 6000 . For unity of presentation, all results refer to time $t_{d}$ based on the major axis. The grid system $p \times q$ is taken to be $128 \times 200$. The numerical outer boundary will be set at $\cosh \xi_{R}=37$ ( $R=37$ for the sphere); the surface radial mesh spacing is taken to be of the order of the standard deviation of diffusion over one time step. Grid dependence has been checked carefully against the time step and the numerical outer boundary; little sensitivity is found between cosh $\xi_{R}=37$ and 48 .

Asymptotic results for the ellipsoid are presented in figure $9(a, b)$ and compared to numerical results (with $\Delta t_{d}=0.01$ and 0.02 ) for the total drag coefficient. Other results are obtained by the hybrid vortex method. The value of $\alpha$ in (4.4) is taken to be 2.2. The tolerance of successive iterations is taken to be $4 \times 10^{-4}$ in a discrete $L_{2}{ }^{-}$ norm. Each cycle of computation from (i) to (iv) takes nearly 1.25 CPU seconds on a Convex C1/XP2 Machine. Some results of the sensitivity analysis for the time step $\Delta t_{d}$ will be presented in figures 3 (sphere) and 9 (ellipsoid). Numerical results at sufficiently long times are presented for the separation angle, wake length, drag coefficient and the streamline pattern. Comparisons for the sphere are made carefully with the previous finite-difference results obtained by Rimon \& Cheng (1969) and with the standard drag curve.

\subsection{Geometric factor $C_{D S} / C_{D F}$}

First, let us examine how the geometry of the ellipsoid biases the contributions of the pressure and the friction to the total drag coefficient. Figure 2 shows the drag ratio $C_{D S} / C_{D F}$ for a wide range of ellipsoid axis ratios according to (2.25). The drag ratio decreases from 1.5 for the sphere to 1 for ellipsoids with large axis ratios. In other words, the contribution of the pressure to $C_{D S}$ decreases from $0.5 C_{D F}$ to 0 as a sphere shrinks on its lateral side to an ellipsoid of small section. Or, we may say that the pressure reinforces the contribution of surface vorticity to the total drag coefficient for bluff bodies. Notice that the pressure also contributes to the volume drag coefficient $C_{D V}$ when there is substantial vorticity contained within the flow. The importance of geometry is fully reflected by the harmonic function $\phi=\phi(x)$; the ratio $C_{D S} / C_{D F}$ is thus a geometric factor. This factor could be the most important connection between the two decompositions: $C_{D}=C_{D F}+C_{D P}$ and $C_{D}=C_{D S}+C_{D V}$ $\left(C_{D A}=0\right.$ in the present study).

\subsection{Results for the sphere}

Figure $3(a, b)$ shows the drag coefficients computed with approaches $\mathbf{S}, \mathbf{P}$ and $\mathrm{V}$ for $R e_{d}=3000$. Except initially, the agreement between the three curves is quite good, especially between $\mathbf{P}$ and $\mathbf{V}$. Approach $\mathbf{S}$, which even exhibits small fluctuations at $R e_{d}=3000$, appears to be rather sensitive to the time step, and thus the grid. This is understandable because the vorticity is not updated solely by finite difference in the present study. These results tend to indicate that one should take paths normal, 


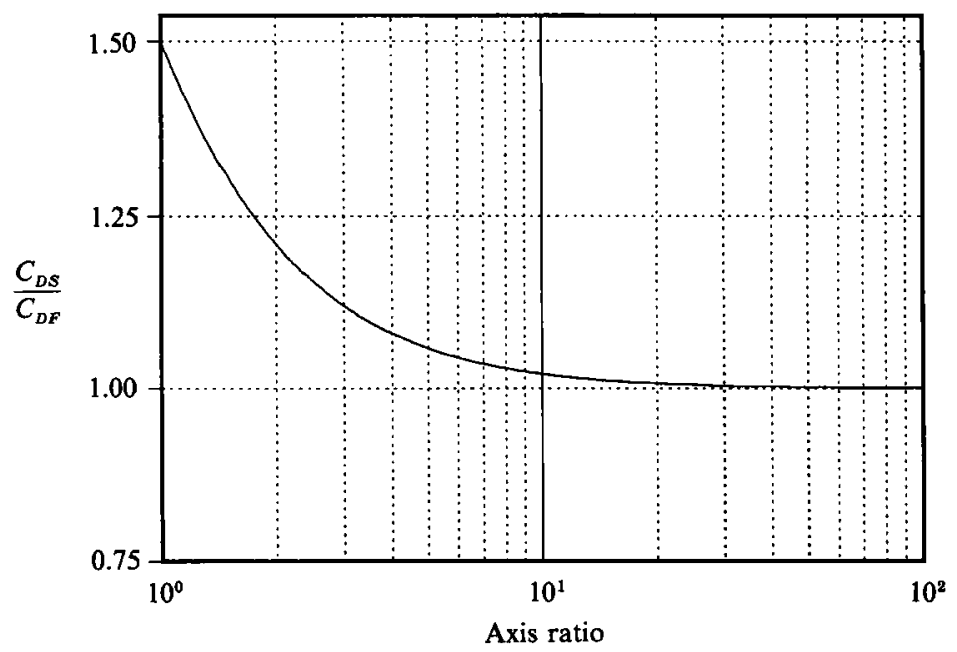

Figure 2. Ratio of $C_{D S}$ to $C_{D F} v s$. the axis ratio of the ellipsoid.
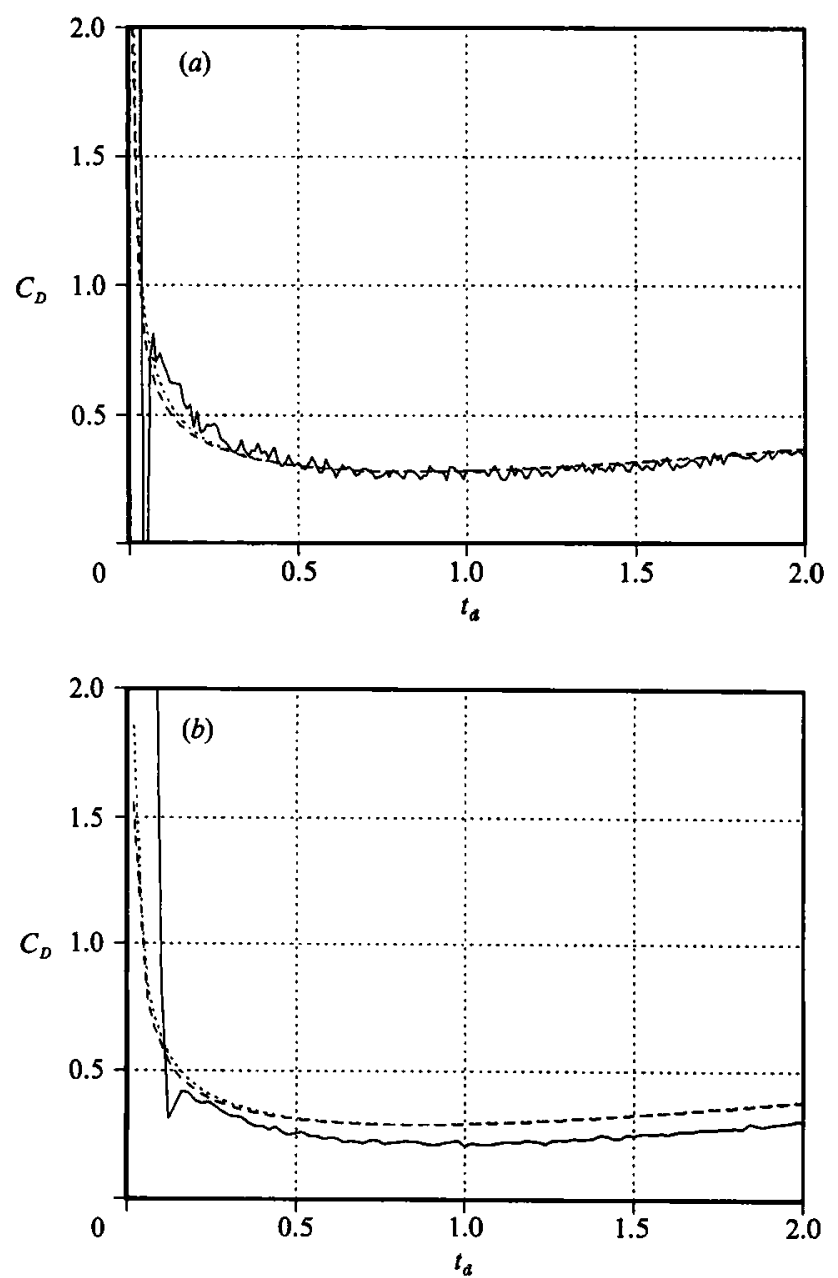

Figure 3. Computed drag coefficients with,$- \mathrm{S} ;---, \mathrm{P}$; and $\cdots, \mathrm{V}$, for $R e_{d}=3000$ : (a) $\Delta t_{d}=0.01$, (b) $\Delta t_{d}=0.02$ (sphere). 

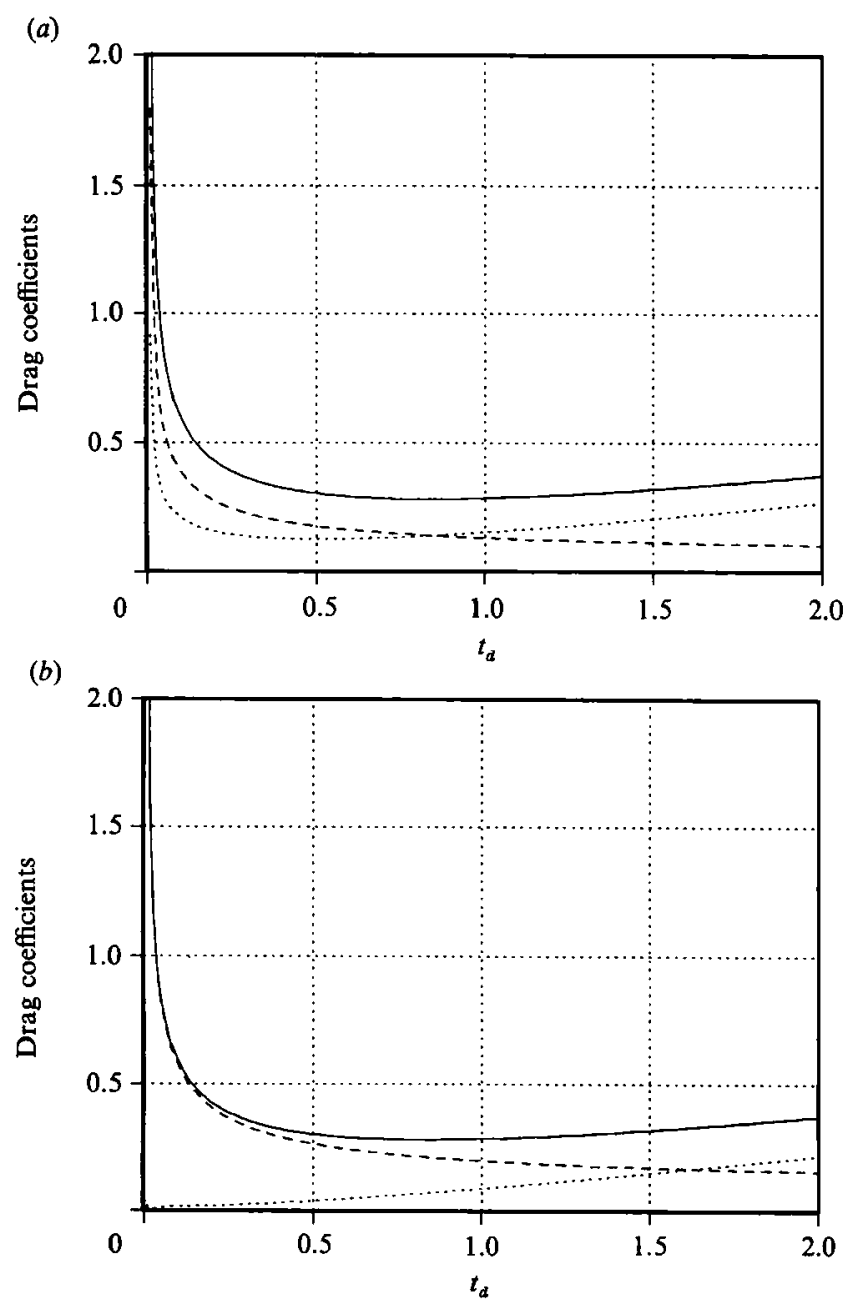

Fraure 4. Time histories of various drag coefficients for $R e_{d}=3000$ for two decompositions : (a) $\longrightarrow, C_{D} ;-\cdots, C_{D F} ; \cdots, C_{D P} ;(b)-, C_{D} ;---, C_{D S} ; \cdots, C_{D V}$ (sphere).

when close, to the body surface like $P$ rather than along the body surface like S. Approximating the unsteady term $\partial u / \partial t$ appears to be easier than approximating the normal derivative $\partial \omega / \partial \xi$ as regards the accuracy. We will therefore take approach $\mathbf{P}$ or $\mathbf{V}$ as our standard method for computing the various drag coefficients.

Figure $4(a, b)$ shows time histories of the drag coefficients for $R e_{d}=3000$ for the two decompositions $C_{D}=C_{D F}+C_{D P}$ and $C_{D}=C_{D S}+C_{D V}$, using $\Delta t_{d}=0.01$. Among the five coefficients involved, it suffices to know any two of them since the geometric factor $C_{D S} / C_{D F}(=1.5)$ is known. Evidently $C_{D V}$, though greater than 0 , is negligible for an initial portion of time since there is no substantial vorticity within the flow; $C_{D}$ is therefore approximately equal to $C_{D S}$. With respect to vorticity, $C_{D S}$ rather than $C_{D F}$ maximizes the usefulness of the surface vorticity as regards the total drag coefficient. Note that the ratio $C_{D V} / C_{D S}$ for flow at higher Reynolds number flow increases with time faster than that at lower Reynolds number.

Figure $5(a-d)$ illustrates four basic flow characteristics: separation time $t_{\mathrm{S}}$, separation angle $\theta_{\mathrm{S}}$, wake length $L=L^{*} / 2 a$ (where $L^{*}$ denotes the dimensional wake length) and the 'stationary' drag coefficient. The results for $t_{\mathrm{s}}$ are refined with 

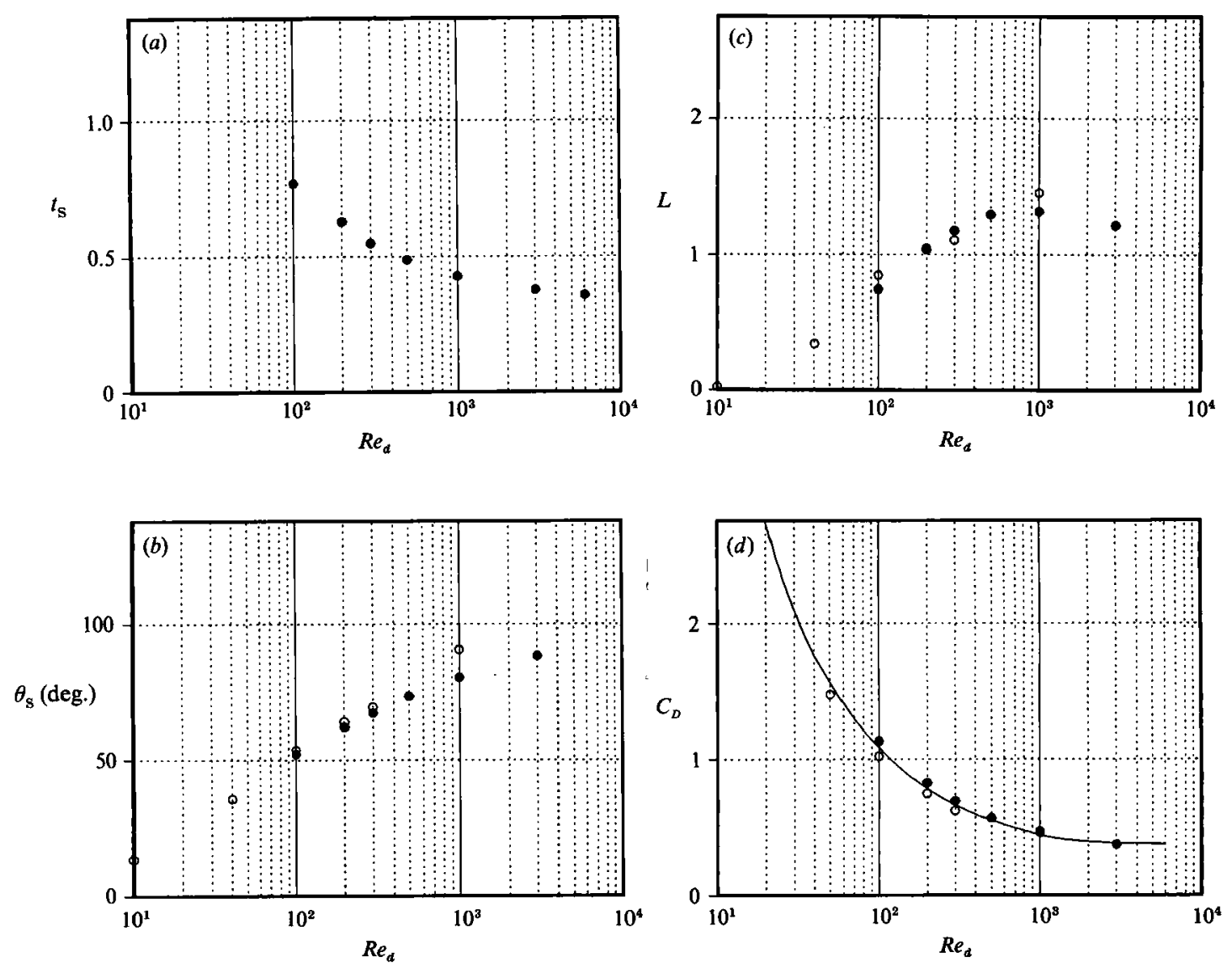

Figure 5. Basic flow characteristics: 0 , present study; $\bigcirc$, Rimon \& Cheng: (a) separation times, (b) separation angles at $t_{d}=32,(c)$ wake lengths at $t_{d}=32,(d)$ drag coefficients at $t_{d}=32$ (sphere).

$\Delta t_{d}=0.005$ while other three flow characteristics are taken at $t_{d}=32$ with $\Delta t_{d}=0.04$. For each case, we terminate the calculation at $t_{d}=32$ (but at $t_{d}=40$ for $R e_{d}=500$, 3000 ). At the time of termination, the flow can hardly be regarded as being steady at $R e_{d}=500$, still less so at $R e_{d}=1000$ and 3000 . For example, we have, for $R e_{d}=$ $500, L=1.3076$ at $t_{d}=25, L=1.3102$ at $t_{d}=32$ and $L=1.3112$ at $t_{d}=40$. Though the increase is only slight, the present study gives no indication that the flow for $R e_{d} \geqslant 500$ has arrived at a steady state by $t_{d}=32$. On the other hand, within the numerical accuracy, it is safe to say that the flows for $R e_{d} \leqslant 300$ have reached their steady states by $t_{d}=32$. Figure $5(a)$ shows that the separation time $t_{\mathrm{s}}$ decreases steadily as the Reynolds number increases, from 0.77 at $R e_{d}=100$ to 0.36 at $R e_{d}=$ 6000 . Figure $5(b)$ shows that the separation angle (at $t_{d}=32$ ) increases with the Reynolds number from $52^{\circ}$ at $R e_{d}=100$ to $88^{\circ}$ at $R e_{d}=3000$. The agreement with Rimon \& Cheng (1969) is generally good but substantial discrepancy is found at $R e_{d}=1000$. Figure $5(c)$ shows a comparison between the present results and the results of Rimon \& Cheng for the wake length. The agreement is quite good at $R e_{d}=200$ and reasonable at $R e_{d}=300$ and 100 , while a larger difference is found at $R e_{d}=1000$. Rimon \& Cheng's results indicate that the wake length increases with the Reynolds number at least in the range from $R e_{d}=10$ to 1000 . The present results, on the contrary, show a maximum at $R e_{d} \approx 1000$ (based on data at $t_{d}=32$ ). Rimon 
(a)

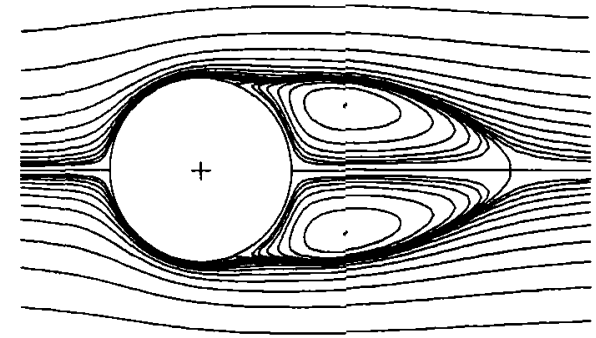

(c)

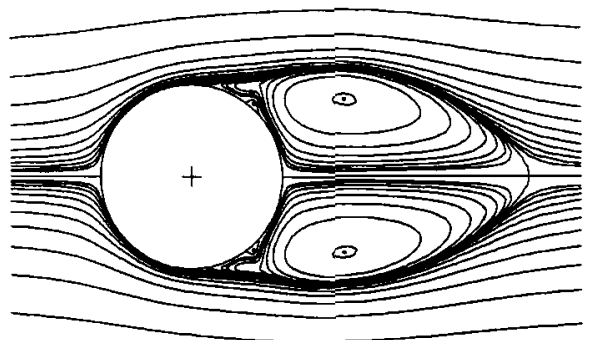

(b)

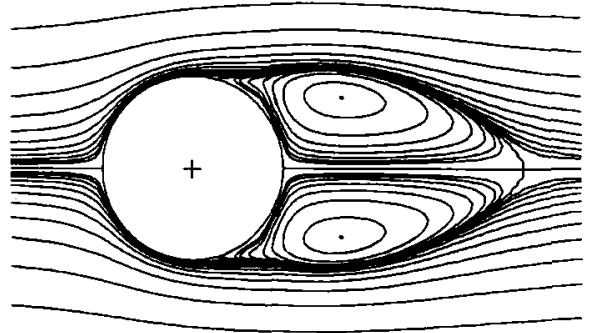

(d)

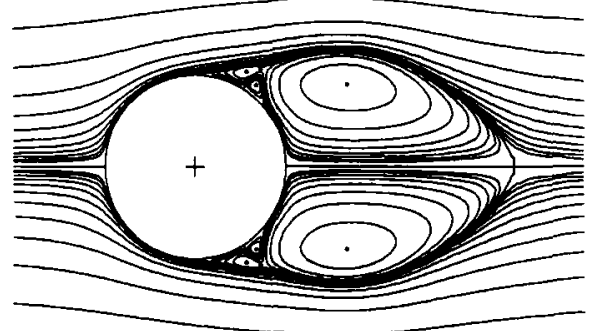

Figure 6. Streamline patterns at $t_{d}=32:(a) R e_{d}=300,(b) 500,(c) 1000,(d) 3000$ (sphere).

\& Cheng's result at $R e_{d}=1000$ deviates from the trend of their results from lower Reynolds numbers - possibly caused by numerical diffusion. Since other detailed experimental or numerical results are lacking at this Reynolds number, this remark is not necessarily conclusive. Figure $5(d)$ illustrates the numerical results for the total drag coefficient. Both the present and Rimon \& Cheng's results are in close agreement with the established stationary values (even at $R e_{d}=3000$ ). The drag coefficient for $R e_{d}=3000$ varies slightly from $t_{d}=32$ to 40 with mild oscillations of amplitude about 0.01 .

Figure $6(a-d)$ presents streamline patterns at $t_{d}=32$ for the Reynolds numbers $300,500,1000$ and 3000 respectively. Here we note that our streamline patterns are very close to those obtained by Rimon \& Cheng for $R e_{d}=200$ (not shown) and 300 since the separation angles and wake lengths are in close agreement with each other. Figure $6(c)$ shows a clearly visible secondary vortex at $R e_{d}=1000$; in this regard, our result is also consistent with Rimon \& Cheng's in spite of the difference in the size of the wake. Furthermore, figure $6(d)$ shows a nice structure which corresponds to the $\alpha$-phenomenon observed for the flow around a circular cylinder at about the same Reynolds number (cf. Ta Phuoc Loc \& Bouard 1985). Some information is now available concerning the relationship between the wake length and the Reynolds number. First, we recall that the flow may not have steady-state solutions for $R e_{d} \geqslant 500$; it is therefore necessary to specify the time of reference in order to have reasonable comparison of the wake lengths at different Reynolds numbers. Ta Phuoc Loc (1980), for a circular cylinder, finds that at $t_{d}=6$ the wake length does decrease with the Reynolds number in the range $R e_{d}=300$ and 1000 . It appears reasonable that the wake length for the sphere may start to decrease at a certain Reynolds number provided the time of reference is given. On the other hand, the separation 
(a)

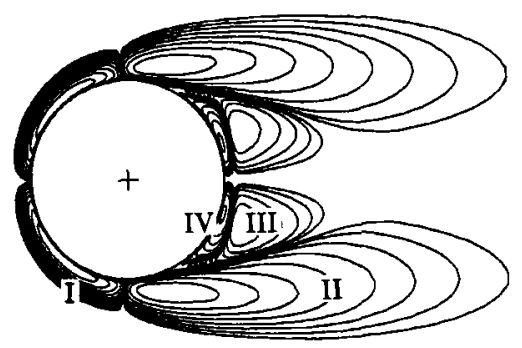

(b)

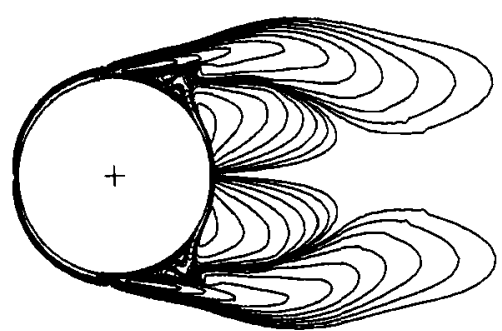

Figure 7. Contours of volume drag elements $D_{\mathrm{e}}(x)$ at $t_{d}=32:$ (a) $R e_{d}=300$, (b) 3000 $\left(D_{\mathrm{e}}= \pm 0.002, \pm 0.004, \pm 0.008, \pm 0.016, \pm 0.032, \pm 0.064, \pm 0.128, \pm 0.256, \pm 0.512, \pm 1.024\right)$ (sphere).

angle does indeed increase with the Reynolds number (cf. figure $5 b$ ); the wake region is actually wider on the lateral sides at higher Reynolds number. The wake length is not necessarily an absolute measure of the wake size. Since secondary vortices appear only at relatively high Reynolds number, we may conclude that the wake region is much more active at higher Reynolds number rather than that the wake length has to increase with the Reynolds number.

Figure $7(a, b)$ presents contour plots of the drag element $D_{\mathrm{e}}(x)$ defined by $(2.26)$ for $R e_{d}=300$ and 3000 . Notice that though the primary vortex is quite large, the region of positive vorticity is effectively confined to a region near the wall (cf. Tritton 1988 , p. 142). Moreover, a calculation within (2.24) reveals that $D_{\mathrm{e}}(x)$ decays like $1 / \cosh ^{2} \xi$ as $\xi \rightarrow \infty$; far-field vorticity therefore contributes little to the drag coefficient. The regions of positive elements $\left(D_{\mathrm{e}}(x)>0\right)$ are basically more intensive or larger than the regions of negative elements $\left(D_{\mathrm{e}}(\boldsymbol{x})<0\right)$. The overall effect is that $C_{D V}>0$ (cf. table 1). One question arises naturally: how are the regions divided? First, from (2.26) we see that one dividing line (actually a surface) must pass through the centre of a vortex since fluid near the centre forms closed streamlines : the vector $\boldsymbol{u} \times \boldsymbol{\omega}$ makes a right angle twice with the smooth field $\boldsymbol{\nabla} \phi$ as one goes around a loop enclosing the centre of a vortex (cf. figure 8 ). Secondly, $D_{\mathrm{e}}(x)$ could most possibly change its sign whenever the vorticity changes its sign. These answers, although partial, are adequate for explaining the observed distribution patterns of drag elements. For example, the contour plot for $R e_{d}=300$ can be divided into four regions, denoted by $\mathrm{I}, \mathrm{II}, \mathrm{III}$ and IV in figure $7(a)$. A similar division applies to the near-wall secondary structure at $R e_{d}=3000$. The sign of I and III is negative while 
(a)

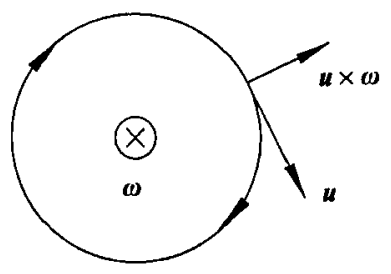

(b)

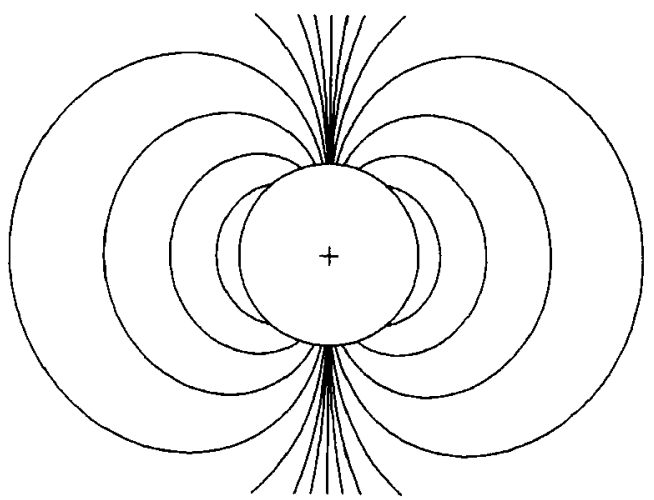

Frgure 8. Schematic of the sign of the drag element $D_{\mathrm{e}}(x):(a)$ flow near the centre,

(b) equipotential line of $\phi$ (sphere).

$\begin{array}{rccccc}R e_{d} & C_{D} & C_{D F} & C_{D P} & C_{D S} & C_{D V} \\ 100 & 1.133 & 0.609 & 0.524 & 0.913 & 0.220 \\ 200 & 0.824 & 0.391 & 0.433 & 0.587 & 0.237 \\ 300 & 0.690 & 0.299 & 0.391 & 0.449 & 0.241 \\ 500 & 0.564 & 0.212 & 0.352 & 0.318 & 0.246 \\ 1000 & 0.457 & 0.133 & 0.324 & 0.200 & 0.257 \\ 3000 & 0.370 & 0.067 & 0.303 & 0.100 & 0.270\end{array}$

TABLE 1. Stationary values of drag coefficients at $t_{d}=32$ (sphere)

the sign of II and IV is positive. Regions II, III are divided by a line passing through the centre of the primary vortex while regions III, IV are divided owing to the sign change of the vorticity. The division of regions I, II is also due to right angles made by the vector $u \times \omega$ with $\nabla \phi$ (cf. figure 8). The upshot of these observations is that effective drag reduction is due to the front region I, but especially region III which is actually the recirculation part of the primary vortex exterior to the wall region IV of positive vorticity. The last fact is physically reasonable concerning the recirculation direction - flow toward the ellipsoid. Recirculation thus accounts, at least partially, for why the drag coefficient $C_{D V}$ or $C_{D}$ may remain fairly stationary while the wake is still growing.

Table 1 lists the values of various drag coefficients at $t_{d}=32$. Contrary to the early stages, the flow contributes significantly to the total drag coefficient $C_{D}$ through $C_{D V}$ which consists of the volume elements $D_{\mathrm{e}}(\boldsymbol{x})$. The ratio of $C_{D V}$ to $C_{D S}$ increases rapidly with the Reynolds number from 0.241 at $R e_{a}=100$ to 2.70 at $R e_{d}=3000$, while the ratio of $C_{D S}$ to $C_{D}$ becomes relatively smaller at higher Reynolds numbers. Evidently, the ratio of $C_{D V}$ to $C_{D S}$ serves as a measure of how active the flow is with vorticity or vortices.

\subsection{Results for the ellipsoid}

Since all basic points have been addressed for the sphere, the discussion here will be brief with particular attention to the differences between the two cases.

Figure $9(a, b)$ shows the drag coefficients obtained from the asymptotic analysis (cf. (3.42)) and computed by the hybrid vortex method with approaches $\mathrm{S}, \mathrm{P}$ and $\mathrm{V}$ for $R e_{d}=3000$. We observe that the asymptotic results are in very close agreement 

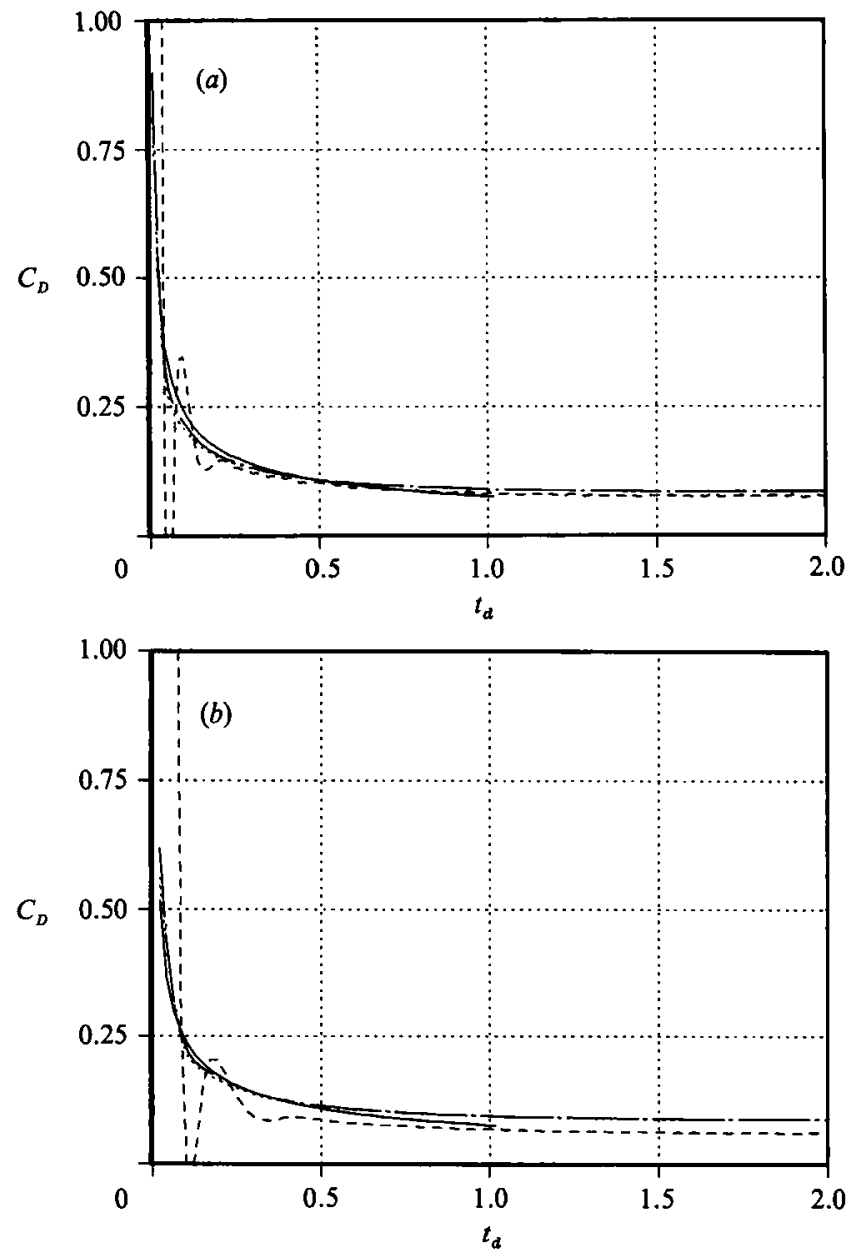

FIGURE 9. Comparisons of asymptotic and computed drag coefficients: - , asymptotic; ,$--- \mathrm{S} ; \cdots, \mathrm{P} ;-\cdot-, \mathrm{V}$ for $R e_{d}=3000:(a) \Delta t_{d}=0.01,(b) 0.02$ (ellipsoid).

with the results obtained with $\mathrm{P}$ and $\mathrm{V}$ even at small times. Approach $\mathrm{S}$, again, exhibits discrepancies from the other two approaches at small times and is sensitive to the time step. These results indicate that even with $\Delta t_{d}=0.02$ the present hybrid vortex method along with $\mathrm{P}$ or $\mathrm{V}$ resolves initial flows with appealing accuracy.

Figure $10(a, b)$ shows time histories of the drag coefficients for $R e_{d}=3000$ in the two decompositions $C_{D}=C_{D F}+C_{D P}$ and $C_{D}=C_{D S}+C_{D V}$, using $\Delta t_{d}=0.01$. Clearly $C_{D S}$ is, again, dominant in the early stages. For the axis ratio $2: 1$, the geometric factor is about 1.21 as can be seen from figure 2. Again, it is remarked that $C_{D V} / C_{D S}$ for flow at higher Reynolds number increases with time faster than at lower Reynolds number. Evidently, all of the various drag coefficients are much smaller than their corresponding values for the sphere in the early stages of flow development.

Figure $11(a-d)$ illustrates four basic flow characteristics: separation time $t_{\mathrm{s}}$, separation angle $\theta_{\mathrm{S}}$, wake length $L$ and the total drag coefficient. The results for $t_{\mathrm{S}}$ are refined with the time step $\Delta t_{d}=0.005$ while the other three flow characteristics are taken at $t_{d}=25$ with $\Delta t_{d}=0.04$. At $t_{d}=25$, the flow appears to reach a steady state for the cases of $R e_{d} \leqslant 500$. Figure $11(a)$ shows that $t_{\mathrm{S}}$ decreases steadily as Reynolds number increases, from 0.51 at $R e_{d}=200\left(1.76\right.$ for $\left.R e_{a}=100\right)$ to 0.11 at $R e_{a}=6000$. 

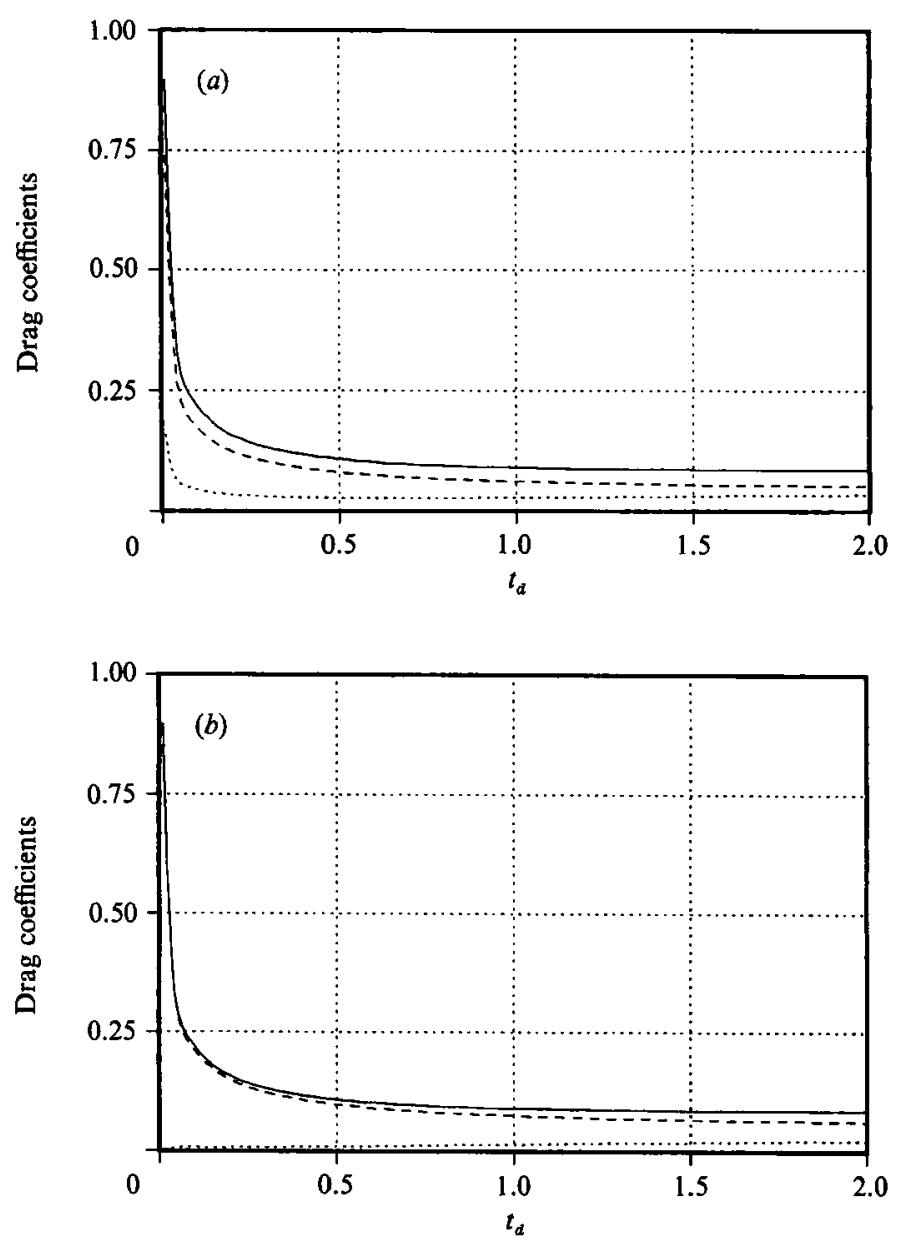

Figure 10. Time histories of various drag coefficients for $R e_{d}=3000$ for two decompositions: $(a)-, C_{D} ;--, C_{D F} ; \cdots, C_{D P} ;(b)-, C_{D} ;---, C_{D S} ; \cdots, C_{D V}$ (ellipsoid).

It is surprising to find that the flow may separate earlier than the corresponding one for the sphere at relatively high Reynolds numbers; this can only be ascribed to the difference in geometry. Figure $11(b)$ shows that the separation angle $\theta_{\mathrm{s}}$ increases with the Reynolds number from $11^{\circ}$ at $R e_{d}=100$ to $67^{\circ}$ at $R e_{d}=6000$. Figure $11(\mathrm{c})$ shows that, as for the sphere, the wake length attains a maximum at about $R e_{d}=3000$ (based on data at $t_{d}=25$ ). Figure $11(d)$ illustrates the numerical results for the total drag coefficient $C_{D}$ : it drops significantly from $0.46(1.133)$ at $R e_{d}=100$ to 0.079 (0.37) at $R e_{d}=3000$; the corresponding values for the sphere are indicated in the parentheses.

Table 2 lists the values of the various drag coefficients at $t_{d}=25$. Relatively smaller contributions come from $C_{D V}$ compared to the cases for the sphere since separation is weaker for the ellipsoid. Notice that the value of $C_{D V}$ never exceeds that of $C_{D S}$ in the range under study, while the sphere has $C_{D V} / C_{D S}$ equal to 1.28 for $R e_{a}=1000$ (and even 2.7 at $R e_{d}=3000$ ). Figure $12(a-d)$ presents four plots of nearly stationary streamline patterns at different Reynolds numbers. Notice that the diameter of the ellipsoids in these plots is larger than that of the spheres in figure 6; the wakes are substantially smaller compared to those for the sphere. A very small, 

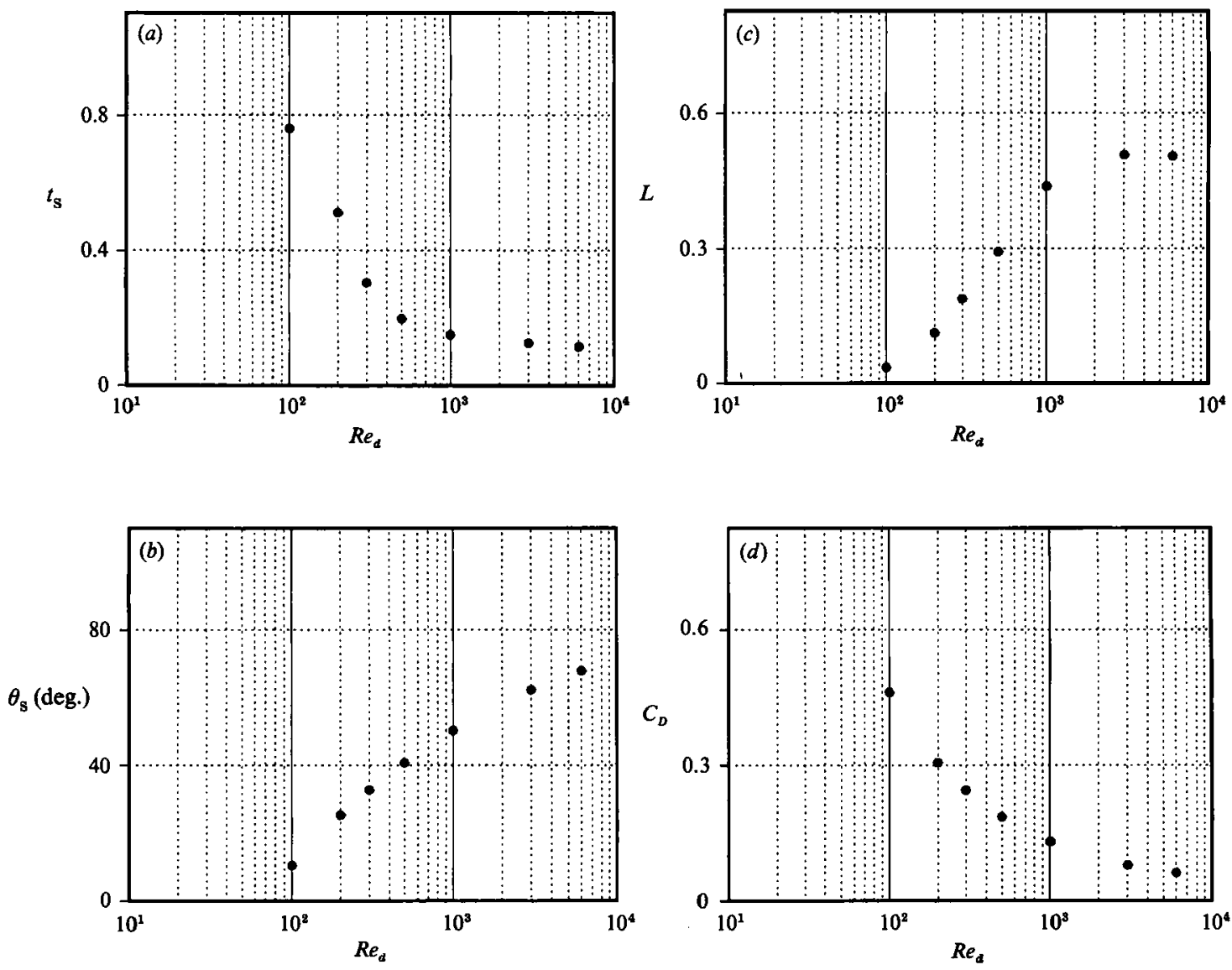

FIGURE 11. Basic flow characteristics: $(a)$ separation times, $(b)$ separation angles at $t_{d}=25$, (c) wake lengths at $t_{d}=25,(d)$ drag coefficients at $t_{d}=25$ (ellipsoid).

\begin{tabular}{cccccc}
\hline$R e_{d}$ & $C_{D}$ & $C_{D F}$ & $C_{D P}$ & $C_{D S}$ & $C_{D V}$ \\
100 & 0.460 & 0.356 & 0.104 & 0.431 & 0.029 \\
200 & 0.305 & 0.228 & 0.077 & 0.276 & 0.029 \\
300 & 0.244 & 0.178 & 0.066 & 0.215 & 0.029 \\
500 & 0.185 & 0.130 & 0.055 & 0.157 & 0.028 \\
1000 & 0.130 & 0.086 & 0.044 & 0.104 & 0.026 \\
3000 & 0.079 & 0.045 & 0.034 & 0.054 & 0.025 \\
6000 & 0.062 & 0.030 & 0.032 & 0.036 & 0.026 \\
& TABLE 2. Stationary values of drag coefficients at $t_{d}=25$ (ellipsoid) \\
\hline
\end{tabular}

but visible secondary vortex can be observed at $R e_{d}=3000$. The appearance of the secondary vortex indicates again that the wake region is more active at higher Reynolds number.

Finally, it has to be emphasized that since flow axisymmetry is assumed in the present study, none of our results give any indication about symmetry-breaking in a real flow, which may depart from symmetry even for $R e_{d}>500$. The presents methods of analysis are, however, not restricted to axisymmetric flow; further study of general three-dimensional flow with the hybrid vortex method is prospective. 
(a)

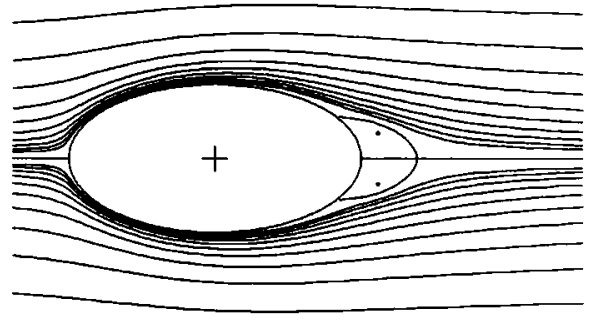

(c)

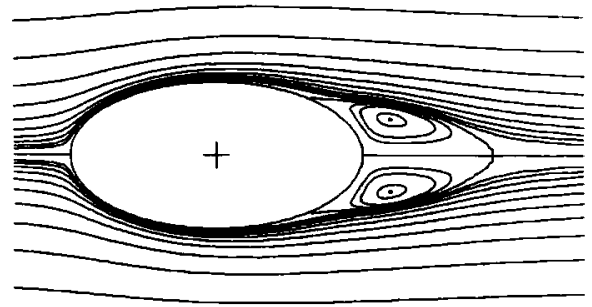

(b)

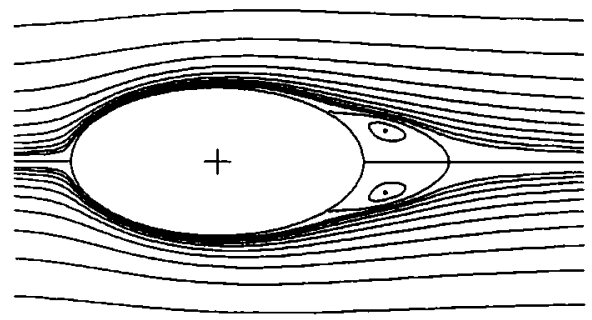

(d)

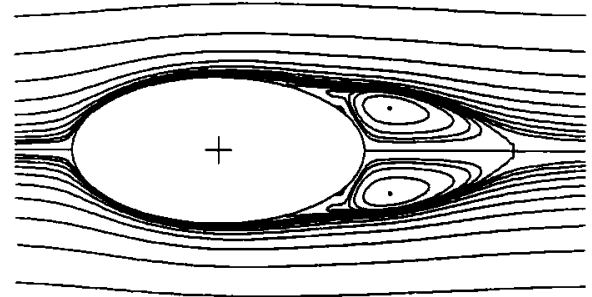

Figure 12. Streamline patterns at $t_{d}=25:(a) R e_{d}=300,(b) 500,(c) 1000,(d) 3000$ (ellipsoid).

\section{Concluding remarks}

The hybrid vortex method is successfully developed for axisymmetric flow around ellipsoids where the stretching effect is present. Comparisons of asymptotic and numerical results at small times validate simultaneously the matched asymptotic analysis and the numerical method. Numerical considerations indicate that it is wise to compute the drag coefficient with approaches $\mathbf{P}$ or $\mathrm{V}$ rather than with $\mathrm{S}: \mathbf{P}$ takes paths normal, when close, to the body while $S$ takes a path along the body surface. Decomposition of the drag coefficient into surface-vorticity and volume-vorticity contributions proves to be appealing in assessing the effect of individual vortex structures in the flow.

The authors thank Professor C. C. Chu of the Institute for his enthusiasm about this work, and for his helpful comments on a draft of this paper. The work is supported in part by the National Science Council of R.O.C. under Contracts NSC790401-E002-25 and NSC80-0401-E002-31.

\section{REFERENCES}

BAR-Lev, M. \& YANG, H. T. 1975 Initial flow field over an impulsively started circular cylinder. J. Fluid Mech. 72, 625.

Batchelor, G. K. 1967 An Introduction to Fluid Dynamics. Cambridge University Press.

Beale, J. T. \& MaJda, A. 1981 Rates of convergence for viscous splitting of the Navier-Stokes equations. Maths Comput. 37, 243.

BEALE, J. T. \& MAJDA, A. 1985 High order accurate vortex methods with explicit kernels. J. Comput. Phys. 58, 188.

Blasıus, H. 1908 Grenzschichten in Flüssigkeiten mit kleiner Reibung. Z. für Math. Phys. 56, 1. 
Chang, C.-C. 1989 Vortex methods and flow around a circular cylinder. In Numerical Methods in Laminar and Turbulent Flow, vol. 6 (ed. C. Taylor, P. Gresho, R. Sani \& J. Häuser), p. 691. Pineridge.

Chang, C.-C. \& Chern, R.-L. 1991 A numerical study of flow around an impulsively started circular cylinder by a deterministic vortex method. J. Fluid Mech. 233, 243.

Chester, W. \& Breach, D. R. 1969 On the flow past a sphere at low Reynolds number. J. Fluid Mech. 37, 751.

Christiansen, J. P. 1973 Numerical simulation of hydrodynamics by the method of point vortices. J. Comput. Phys. 13, 363.

Dennis, S. C. R. \& Walker, J. D. A. 1972 Numerical solutions for time-dependent flow past an impulsively started sphere. Phys. Fluids 15, 517.

HALD, O. H. 1979 The convergence of vortex methods II. SIAM J. Numer. Anal. 16, 726.

Hamielec, A. E., Hoffman, T. W. \& Ross, L. L. 1967 Numerical solution of the Navier-Stokes equation for flow past spheres. Parts I and II. AIChE J. 13, 212.

Kim, S. \& Arunachalam, P. V. 1987 The general solution for an ellipsoid in low-Reynoldsnumber flow. J. Fluid Mech. 178, 535.

Landau, L. D. \& Lifshitz, E. M. 1987 Fluid Mechanics (2nd Edn). Pergamon.

LeClair, B. P., Hamielec, A. E. \& Pruppacher, H. R. 1970 A numerical study of the drag on a sphere at low and intermediate Reynolds numbers. J. Atmos. Sci. 27, 308.

Oliver, D. L. R. \& Chung, J. N. 1985 Steady flows inside and around a fluid sphere at low Reynolds numbers. J. Fluid Mech. 154, 215.

Peace, A. J. \& Riley, N. 1983 A viscous vortex pair in ground effect. J. Fluid Mech. 129, 409.

QuaRtapelie, L. \& Napolitano, M. 1983 Force and moment in incompressible flows. AIAA J. $21,911$.

Rimon, Y. \& Cheng, S. I. 1969 Numerical solution of a uniform flow over a sphere at intermediate Reynolds numbers. Phys. Fluids 12, 949.

Sano, T. 1981 Unsteady flow past a sphere at low Reynolds number. J. Fluid Mech. 112, 433.

SARPKAYA, T. 1989 Computational method with vortices - the 1988 Freeman Scholar Lecture. Trans. ASME I: J. Fluids Engng 111, 5.

TA PHUoc Loc 1980 Numerical analysis of unsteady secondary vortices generated by an impulsively started circular cylinder. J. Fluid Mech. 100, 111.

Ta Phuoc Loc \& Bouard, R. 1985 Numerical solution of the early stage of unsteady viscous flow around a circular cylinder : a comparison with experimental visualization and measurements. J. Fluid Mech. 160, 93.

Tritton, D. J. 1988 Physical Fluid Dynamics (2nd Edn). Oxford University Press.

Van Dyke, M. 1975 Perturbation Methods in Fluid Mechanics. Parabolic.

WANG, C. Y. 1967 Separation and stall of an impulsively started elliptic cylinder. Trans. ASME C: J. Appl. Mech. 34, 823. 